\title{
@creative
}

Also available at http://amc-journal.eu

ISSN 1855-3966 (printed edn.), ISSN 1855-3974 (electronic edn.)

ARS MATHEMATICA CONTEMPORANEA 12 (2017) 383-413

\section{Vertex-transitive graphs and their arc-types}

\author{
Marston D. E. Conder * \\ Department of Mathematics, University of Auckland, Auckland, New Zealand
}

Tomaž Pisanski ${ }^{\dagger}$

University of Primorska, Koper and IMFM, Ljubljana, Slovenia

Arjana Žitnik

University of Ljubljana and IMFM, Ljubljana, Slovenia

Received 30 June 2016, accepted 24 December 2016, published online 12 February 2017

\begin{abstract}
Let $X$ be a finite vertex-transitive graph of valency $d$, and let $A$ be the full automorphism group of $X$. Then the arc-type of $X$ is defined in terms of the sizes of the orbits of the stabiliser $A_{v}$ of a given vertex $v$ on the set of arcs incident with $v$. Such an orbit is said to be self-paired if it is contained in an orbit $\Delta$ of $A$ on the set of all arcs of $X$ such that $\Delta$ is closed under arc-reversal. The arc-type of $X$ is then the partition of $d$ as the sum $n_{1}+n_{2}+\cdots+n_{t}+\left(m_{1}+m_{1}\right)+\left(m_{2}+m_{2}\right)+\cdots+\left(m_{s}+m_{s}\right)$, where $n_{1}, n_{2}, \ldots, n_{t}$ are the sizes of the self-paired orbits, and $m_{1}, m_{1}, m_{2}, m_{2}, \ldots, m_{s}, m_{s}$ are the sizes of the non-self-paired orbits, in descending order. In this paper, we find the arc-types of several families of graphs. Also we show that the arc-type of a Cartesian product of two 'relatively prime' graphs is the natural sum of their arc-types. Then using these observations, we show that with the exception of $1+1$ and $(1+1)$, every partition as defined above is realisable, in the sense that there exists at least one vertex-transitive graph with the given partition as its arc-type.
\end{abstract}

Keywords: Symmetry type, vertex-transitive graph, arc-transitive graph, Cayley graph, Cartesian product, covering graph.

Math. Subj. Class.: 05E18, 20B25, 05C75, 05C76

\footnotetext{
${ }^{*}$ This work was supported in part by the N.Z. Marsden Fund (via grant UOA1323).

${ }^{\dagger}$ Research supported in part by the ARRS (via grants P1-0294, N1-0032, L7-5554, J1-7051, J1-6720).

${ }^{\ddagger}$ Research supported in part by the ARRS (via grant P1-0294) and the European Science Foundation (Eurocores Eurogiga, GReGAS (N1-0011))

E-mail addresses: m.conder@auckland.ac.nz (Marston D. E. Conder), tomaz.pisanski@upr.si (Tomaž Pisanski), arjana.zitnik@fmf.uni-lj.si (Arjana Žitnik)
}

(a) (i) This work is licensed under http://creativecommons.org/licenses/by/3.0/ 


\section{Introduction}

Vertex-transitive graphs hold a significant place in mathematics, dating back to the time of first recognition of the Platonic solids, and also now in other disciplines where symmetry (and even other properties such as rigidity) play an important role, such as fullerene chemistry, and interconnection networks.

A major class of vertex-transitive graphs is formed by Cayley graphs, which represent groups in a very natural way. (For example, the skeleton of the $C_{60}$ molecule is a Cayley graph for the alternating group $A_{5}$.) It is relatively easy to test whether a given vertextransitive graph is a Cayley graph for some group: by a theorem attributed to Sabidussi [23], this happens if and only if the automorphism group of the graph contains a subgroup that acts regularly on vertices. Vertex-transitive graphs that fail this test are relatively rare, the Petersen graph being a famous example. A recent study of small vertex-transitive graphs of valency 3 in [18] shows that among 111360 such graphs of order up to 1280, only 1434 of them are not Cayley graphs.

For almost every Cayley graph, the automorphism group itself acts regularly on vertices (see [1]). Any such graph is called a graphical regular representation of the group $G$, or briefly, a GRR. In a book by Coxeter, Frucht and Powers [8] devoted to the study of 3valent GRRs, vertex-transitive 3-valent graphs were classified into four types, according to the action of the automorphism group on the arcs (ordered pairs of adjacent vertices) of the graph. One class consists of those graphs which are arc-transitive, another of those for which there are two orbits on arcs, and the other two are two classes of GRRs. Arctransitive graphs are also called symmetric.

Symmetric graphs have been studied quite intensively, especially in the 3 -valent case, by considering the action of the automorphism group on non-reversing walks of given length $s$, known as $s$-arcs. For example, it was shown by Tutte [26, 27] that every finite symmetric 3-valent graph is $s$-arc-regular for some $s \leq 5$, and hence that the order of the stabiliser of a vertex in the automorphism group of every such graph is bounded above by 48. Tutte's theorem and related work have been used to determine all symmetric 3 -valent graphs on up to 10, 000 vertices; see [9, 6, 5]. Also Tutte's seminal theorem was generalised much later by Weiss, who used the classification of doubly-transitive permutation groups to prove that every finite symmetric graph of valency greater than 2 is $s$-arc-transitive but not $(s+1)$-arc-transitive for some $s \leq 7$, and in particular, that there are no 8-arc-transitive finite graphs; see [29].

Another important class of vertex-transitive graphs was investigated by Tutte [28] and Bouwer [3], namely the graphs that are vertex- and edge-transitive but not arc-transitive. These are now called half-arc-transitive graphs. Every such graph has even valency, and its automorphism group has two orbits on arcs, with every $\operatorname{arc}(v, w)$ and its reverse $(w, v)$ lying in different orbits; see [28, p. 59]. Bouwer [3] constructed a family of examples containing one half-arc-transitive graph of each even valency greater than 2 , and the first and third authors of this paper have recently proved that other examples of the type considered by Bouwer produce infinitely many of every such valency; see [7].

According to Coxeter, Frucht and Powers [8], the idea of classifying cubic vertextransitive graphs with respect to the arc-orbits of the automorphism group originated in some work by Ronald Foster over half a century ago, which was presented to his friends in the form of unpublished notes. The classification is carried out rigorously in their book [8]. Although Foster's original idea of 'zero-symmetric' graphs was later expanded to other valencies under the term GRR, the classification by arc-orbits itself was never extended 
in a systematic way to graphs of other valencies. This paper provides a remedy for that omission. By introducing the concept of 'arc-type', we provide a language that can be used to unify the notions of arc-transitivity and half-arc-transitivity and the above-mentioned classification of symmetric 3 -valent graphs, and also to extend this classification to vertextransitive graphs of higher valency.

We can now define the notion of arc-type for a vertex-transitive graph. Let $X$ be a $d$-valent vertex-transitive graph, with automorphism group $A$. We first make a critical observation about the pairing of arc-orbits. The orbit of an $\operatorname{arc}(v, w)$ under the action of $A$ can be paired with the orbit of $A$ containing the reverse arc $(w, v)$, and if these orbits are the same, then the given orbit is said to be self-paired. This is similar to the definition of paired orbitals for transitive permutation groups. But here we will abuse notation and extend the definition to the orbits of the stabiliser $A_{v}$ in $A$ of a vertex $v$ on the arcs emanating from $v$, and say that the orbit of $A_{v}$ containing the arc $(v, w)$ is self-paired if $(v, w)$ lies in the same orbit of $A$ as its reverse $(w, v)$.

We define the arc-type of $X$ as the partition $\Pi$ of $d$ as the sum

$$
\Pi=n_{1}+n_{2}+\cdots+n_{t}+\left(m_{1}+m_{1}\right)+\left(m_{2}+m_{2}\right)+\cdots+\left(m_{s}+m_{s}\right)
$$

where $n_{1}, n_{2}, \ldots, n_{t}$ are the sizes of the self-paired arc-orbits of $A_{v}$ on the arcs emanating from $v$, and $m_{1}, m_{1}, m_{2}, m_{2}, \ldots, m_{s}, m_{s}$ are the sizes of the non-self-paired arc-orbits, in descending order.

Similarly, the edge-type of $X$ is the partition of $d$ as the sum of the sizes of the orbits of $A_{v}$ on edges incident with $v$, and can be found by simply replacing each bracketed term $\left(m_{j}+m_{j}\right)$ by $2 m_{j}$ (for $\left.1 \leq j \leq s\right)$.

The number of possibilities for the arc-type $\Pi$ depends on the valency $d$. For $d=1$ there is just one possibility, namely with $n_{1}=1$, and this occurs for the complete graph $K_{2}$. For $d=2$, in principle there could be three possibilities, namely $2,1+1$ and $(1+1)$, but every 2-valent connected graph is a cycle, and is therefore arc-transitive, with arc-type 2. In particular, $1+1$ and $(1+1)$ cannot occur as arc-types. For $d=3$ there are four possibilities (namely $3,2+1,1+1+1$ and $1+(1+1)$ ), and they all occur, as shown in [8]. A natural question arises as to what arc-types occur for higher valencies.

In this paper, we provide some basic theory for arc-types, which helps us to answer that question. In particular, we give for each positive integer $d$ the number of different partitions of the above form $(\dagger)$ for $d$, by means of a generating function. This gives a closed form solution for the number of different possibilities in the case of a GRR of given valency $d$. (As a curiosity, we mention that there is also a connection with the different root types of polynomials with real coefficients.)

Then our main theorem states that with the exception of $1+1$ and $(1+1)$, every partition $\Pi$ as defined in $(\dagger)$ is realisable, in the sense that there exists at least one vertex-transitive graph with $\Pi$ as its arc-type. To prove this, we consider how to combine 'small' vertextransitive graphs into a larger vertex-transitive graph, preserving (but increasing the number of) the summands in the arc-type. The key step is to show that the arc-type of a Cartesian product of such graphs is just the sum of their arc-types, when the graphs are 'relatively prime' with respect to the Cartesian product.

Our proof of the theorem then reduces to finding suitable 'building blocks', to use as base cases for the resulting construction. Several interesting families and examples of graphs are found to be helpful. In particular, we introduce the concept of a special kind of thickened cover of a graph, obtained by replacing some edges of the given graph by 
complete bipartite graphs, and other edges by ladder graphs (with 'parallel' edges). Under some special conditions, the thickened cover is vertex-transitive, and it is easy to compute its arc-type from the arc-type of the given base graph. Note that this does not work in general. It can happen that a group $G$ acts transitively on the vertices of the graph, with given arc-type, but the full automorphism group is larger than $G$. The challenge is to ensure that no further automorphisms are admitted.

Finally, as a corollary of our main theorem, we show that every standard partition of a positive integer $d$ is realisable as the edge-type of a vertex-transitive graph of valency $d$, except for $1+1$ (when $d=2$ ).

Vertex-transitive graphs are key players in algebraic graph theory, but also (as intimated earlier) they have important applications in other branches of mathematics. In group theory they play a crucial role as Cayley graphs. In geometry they are encountered in convex and abstract polytopes, incidence geometries, and configurations, and in manifold topology they feature in the study of regular and chiral maps and hypermaps, and Riemann and Klein surfaces with large automorphism groups.

Classification of vertex-transitive graphs by their edge- or arc-type gives a new viewpoint, and helps provide a better understanding of their structure. This approach can also be fruitful in terms of determining all small examples of various kinds of graphs, akin to the census of 3 -valent symmetric graphs on up to 10000 vertices $[9,6,5]$, the census of vertextransitive graphs up to 31 vertices [22], or the census of small 4-valent half-arc-transitive graphs and arc-transitive digraphs of valency 2 [20]. For example, the construction used by Potočnik, Spiga and Verret to obtain their census of vertex-transitive 3-valent graphs on up to 1280 vertices in [18] depends on the edge-type, and their census of all connected quartic arc-transitive graphs of order up to 640 (also in [18]) was obtained by associating some of them with vertex-transitive 3 -valent graphs of edge-type $2+1$ (and using cycle decompositions); see also [19,21]. In these cases it was a stratified approach that enabled the limits of the census to be pushed so high, and it is likely that for graphs of higher valency or larger order, this kind of approach will be invaluable.

\section{Preliminaries}

All the graphs we consider in this paper are finite, simple, undirected and non-trivial. Given a graph $X$, we denote by $V(X)$ and $E(X)$ the set of vertices and the set of edges of $X$, respectively. We denote an edge of $X$ with vertices $u$ and $v$ by $\{u, v\}$, or sometimes more briefly by $u v$. We will occasionally use triangle (respectively quadrangle) to denote an unoriented 3-cycle (resp. 4-cycle) in a graph, and will say that two triangles are disjoint if they have no vertex in common. For any vertex $v$ of $X$, we denote by $E(v)$ the set of edges of $X$ incident with $v$. An $\operatorname{arc}$ is an ordered pair of adjacent vertices, and we denote by $A(X)$ the set of all arcs of $X$. Associated with each edge $\{u, v\}$ there are two arcs, which we denote by $(u, v)$ and its reverse $(v, u)$. Also we define $A(v)$ as the set of all $\operatorname{arcs}(v, w)$ of $X$ emanating from a given vertex $v$.

The automorphism group of $X$ is denoted by $\operatorname{Aut}(X)$. Note that the action of $\operatorname{Aut}(X)$ on the vertex-set $V(X)$ also induces an action of $\operatorname{Aut}(X)$ on the edge-set $E(X)$ and one on the arc-set $A(X)$. If the action of $\operatorname{Aut}(X)$ is transitive on the vertex-set, edge-set, or arcset, then we say that $X$ is vertex-transitive, edge-transitive or arc-transitive, respectively. An arc-transitive graph is often also called symmetric. The graph $X$ is half-arc-transitive if it is vertex-transitive and edge-transitive, but not arc-transitive. Note that the valency of 
a half-arc-transitive graph is necessarily even; see [28, p. 59].

In this paper we only consider vertex-transitive graphs. Obviously, vertex-transitive graphs are always regular. Moreover, because a disconnected vertex-transitive graph consists of pairwise isomorphic connected components, we may restrict our attention here to connected graphs. Also we will sometimes use 'VT' as an abbreviation for vertextransitive.

Next, let $G$ be a group, and let $S$ be a subset of $G$ that is inverse-closed and does not contain the identity element. Then the Cayley graph $\operatorname{Cay}(G, S)$ is the graph with vertexset $G$, and with vertices $u$ and $v$ being adjacent if and only if $v u^{-1} \in S$ (or equivalently, $v=x u$ for some $x \in S$ ). Since we require $S$ to be inverse-closed, this Cayley graph is undirected, and since $S$ does not contain the identity, the graph has no loops. Also $\operatorname{Cay}(G, S)$ is regular, with valency $|S|$, and is connected if and only if $S$ generates $G$. Furthermore, it is easy to see that $G$ acts as a group of automorphisms of $\operatorname{Cay}(G, S)$ by right multiplication, and this action is transitive on vertices, with trivial stabiliser, and so this action of $G$ on $\operatorname{Cay}(G, S)$ is sharply-transitive (or regular). Hence in particular, $\operatorname{Cay}(G, S)$ is vertex-transitive.

Indeed the following (which is attributed to Sabidussi [23]) shows how to recognise Cayley graphs:

Lemma 2.1. A graph $X$ is a Cayley graph for the group $G$ if and only if $G$ acts regularly on $V(X)$ as a group of automorphisms. More generally, a graph $X$ is a Cayley graph if and only if some subgroup of $\operatorname{Aut}(X)$ acts regularly on $V(X)$.

Observe that for a fixed $x \in S$, all the edges of form $\{u, x u\}$ and $\left\{u, x^{-1} u\right\}$ for $u \in G$ lie in the same edge-orbit (as each other) under the automorphism group of $\operatorname{Cay}(G, S)$, and similarly, that all the arcs of the form $(u, x u)$ lie in the same arc-orbit. If all such arc-orbits are distinct, then $G$ is the full automorphism group of $\operatorname{Cay}(G, S)$, and $\operatorname{Cay}(G, S)$ is called a graphical regular representation of the group $G$, or briefly a $G R R$ for $G$. Another term for such a graph is zero-symmetric. Note that if $X$ is a connected zero-symmetric graph (or GRR) with valency $d$, then $X$ has $d$ arc-orbits, and all the arcs emanating from a given vertex $v$ lie in different arc-orbits. Moreover, if $G=\operatorname{Aut}(X)$, then the stabiliser $G_{v}$ of any vertex $v$ must fix each neighbour of $v$, and then by connectedness, it follows that $G_{v}$ is trivial. Thus $G$ acts regularly on $V(X)$, and so by Lemma 2.1, the graph $X$ is a Cayley graph for $G$.

Next, we describe another group-theoretic construction, for a special class of vertextransitive graphs. Let $G$ be a group, let $H$ be a subgroup of $G$, and let $a$ be an element of $G$ such that $a^{2} \in H$. Now define a graph $\Gamma=\Gamma(G, H, a)$ by setting

$$
V(\Gamma)=\{H g: g \in G\} \quad \text { and } \quad E(\Gamma)=\left\{\{H x, H y\}: x, y \in G \mid x y^{-1} \in H a H\right\} .
$$

This graph $\Gamma$ is called a (Sabidussi) double coset graph. As with Cayley graphs, the given group $G$ induces a group of automorphisms of $\Gamma(G, H, a)$ by right multiplication, since $(x g)(y g)^{-1}=x g g^{-1} y^{-1}=x y^{-1} \in H a H$ whenever $\{H x, H y\} \in E(\Gamma)$. Again this action is vertex-transitive, since $(H x) x^{-1} y=H y$. Moreover, the stabiliser of the vertex $H$ is $G_{H}=\{g \in G \mid H g=H\}$, which is $H$ itself, and this acts transitively on the neighbourhood $\{H a h: h \in H\}$ of $H$, so in fact $\Gamma(G, H, a)$ is arc-transitive. Conversely, every non-trivial arc-transitive graph $X$ can be constructed in this way, by taking $G=\operatorname{Aut}(X)$, and $H=G_{v}$ for some $v \in V(X)$, and $a$ as any automorphism in $G$ that interchanges $v$ with one of its neighbours. 
Finally, we mention a convenient way to describe cubic Hamiltonian graphs, that will be helpful later. Let $X$ be a cubic Hamiltonian graph on $n$ vertices. Label the vertices of $X$ with numbers $0, \ldots, n-1$, such that vertices $i$ and $i+1$ are consecutive in a given Hamilton cycle for $0 \leq i<n$ (treated modulo $n$ ). Each vertex $i$ is adjacent to $i-1$ and $i+1(\bmod n)$, and to one other vertex, which has label $v_{i}$, say. Now define $d_{i}=v_{i}-i$ for $0 \leq i<n$. Then the $L C F$-code of $X$ is given by the sequence $\left[d_{0}, \ldots, d_{n-1}\right]$. Clearly the LCF-code defines the graph $X$, since the edges are $\{i, i+1\}$ for all $i(\bmod n)$ and $\left\{i, i+d_{i}\right\}$ for all $i(\bmod n)$. On the other hand, the LCF is not necessarily unique for $X$, since it depends on the choice of Hamilton cycle. Note also that if the code sequence is periodic, then it is sufficient to list a sub-sequence and indicate how many times it repeats, using a superscript. For example, $[3,-3]^{4}$ is the LCF-code of a 3-dimensional cube.

\section{Edge-types and integer partitions}

Let $X$ be a vertex-transitive graph of valency $d$, and let $\left\{\Delta_{1}, \ldots, \Delta_{k}\right\}$ be the set of orbits of $G=\operatorname{Aut}(X)$ on $E(X)$. This partition of $E(X)$ into orbits also induces a partition of the set $E(v)$ of all edges incident with a given vertex $v$, namely into the sets $E(v) \cap \Delta_{i}$ for $1 \leq i \leq k$. These are simply the restrictions of the edge-orbits $\Delta_{i}$ to the set $E(v)$.

If we let $\ell_{i}=\left|E(v) \cap \Delta_{i}\right|$ for each $i$, then we may define the edge-type of $X$ to be the partition of the valency $d$ as the sum

$$
\ell_{1}+\ell_{2}+\cdots+\ell_{k}
$$

where we assume the numbers $\ell_{i}$ are in descending order. Note that by vertex-transitivity, the numbers $\ell_{i}$ do not depend on the choice of $v$. (Indeed when $X$ is finite, counting incident vertex-edge pairs $(v, e)$ with $e \in \Delta_{i}$ gives $2\left|\Delta_{i}\right|=|V(X)| \ell_{i}$ and therefore $\ell_{i}=$ $2\left|\Delta_{i}\right| /|V(X)|$ for each $i$.) Hence in particular, the edge-type does not depend on the choice of $v$. We denote the edge-type of $X$ by et $(X)$.

It is not at all obvious as to which partitions can occur as the edge-type of a vertextransitive graph. The edge-type of a vertex-transitive cubic graph can be 3 , or $2+1$, or $1+1+1$, while that of a vertex-transitive quartic graph can be 4 , or $3+1$, or $2+2$, or $2+1+1$, or $1+1+1+1$. We will see instances of all of these in Section 5. Then later, in Section 9, we will show that with the exception of $1+1$ (for $d=2$ ), every standard partition of a given positive integer $d$ can be realised as an edge-type.

To enumerate the possibilities for a given valency $d$, we may use generating functions for integer partitions. Let $p(d, k)$ denote the number of partitions of $d$ with $k$ parts, and let $p(d)$ denote the number of partitions of an integer $d$. Obviously, $p(d)=\sum_{k} p(d, k)$.

The generating functions for integer partitions are very well-known, and can be found in [30, p. 100] for example. In fact, the generating function $P(x, y)$ for $p(d, k)$ is given by

$$
P(x, y)=\sum_{d} \sum_{k \geq 0} p(d, k) y^{k} x^{d}=\prod_{n \geq 1} \frac{1}{1-y x^{n}}
$$

and then by taking $y=1$, we get

$$
P(x)=\prod_{n \geq 1} \frac{1}{1-x^{n}}=\sum_{d \geq 0} p(d) x^{d}
$$

as the generating function for $p(d)$ itself. 


\section{Arc-types and marked partitions}

In this section we refine the notion of edge-type of a vertex-transitive graph $X$, by considering the action of $\operatorname{Aut}(X)$ on the $\operatorname{arcs}$ of $X$.

Let $\Delta$ be an orbit of $\operatorname{Aut}(X)$ on $A(X)$, and let $\Delta^{*}=\{(v, u):(u, v) \in \Delta\}$ be its paired orbit, in the same way that a permutation group on a set $\Omega$ has paired orbitals on the Cartesian product $\Omega \times \Omega$. Note that $\Delta^{*}$ is also an orbit of $\operatorname{Aut}(X)$ on $A(X)$, and that the union $\Delta \cup \Delta^{*}$ consists of all the arcs obtainable from an orbit of $\operatorname{Aut}(X)$ on edges of $X$. We say that $\Delta$ is self-paired if $\Delta=\Delta^{*}$ and non-self-paired if $\Delta \neq \Delta^{*}$.

We can now write the orbits of $\operatorname{Aut}(X)$ on $A(X)$ as

$$
\Delta_{1}, \ldots, \Delta_{t}, \Delta_{t+1}, \Delta_{t+1}^{*}, \ldots, \Delta_{t+s}, \Delta_{t+s}^{*}
$$

where $\Delta_{1}, \ldots, \Delta_{t}$ are self-paired, while $\Delta_{t+1}, \ldots, \Delta_{t+s}$ are non-self-paired.

This partition of $A(X)$ into the orbits of $\operatorname{Aut}(X)$ also induces a partition of the set of arcs emanating from a given vertex. For any vertex $v$ of $X$, define $n_{i}=\left|A(v) \cap \Delta_{i}\right|$ for $1 \leq i \leq t$, and $m_{j}=\left|A(v) \cap \Delta_{j}\right|$ for $t+1 \leq j \leq t+s$. Again since $X$ is vertex-transitive, these numbers do not depend on the choice of $v$, and then furthermore, arc-reversal gives $\left|A(v) \cap \Delta_{j}^{*}\right|=\left|A(v) \cap \Delta_{j}\right|=m_{j}$ for $t+1 \leq j \leq t+s$.

Hence the $n_{i}$ are the sizes of the self-paired arc-orbits restricted to $A(v)$, while the $m_{j}$ are the sizes of the non-self-paired arc-orbits restricted to $A(v)$, and thus we obtain

$$
d=|A(v)|=n_{1}+\cdots+n_{t}+\left(m_{1}+m_{1}\right)+\cdots+\left(m_{s}+m_{s}\right),
$$

just as in $(\dagger)$ in the Introduction. This is the arc-type of $X$, and we denote it by at $(X)$.

We may call the expression on the right-hand-side of the above a marked partition of the integer $d$. By this, we mean simply a partition in which some pairs of equal-valued summands are placed in parentheses. Note that the parentheses in a marked partition $\Pi$ are important, because when $\Pi$ represents the arc-type of a VT graph, they indicate that the two numbers summed between the parentheses are the sizes of two paired arc-orbits corresponding to the same edge-orbit.

Since the order of the arc-orbits of each of the two kinds (self-paired and non-selfpaired) can be chosen arbitrarily, we may consider two marked partitions to be equal if they have the same summands, possibly in a different order. Usually we will assume that the summands of each kind (unbracketed and bracketed) are in descending order, so that $n_{1} \geq \cdots \geq n_{t}$ and $m_{1} \geq \cdots \geq m_{s}$.

In a sense, the three most important classes of vertex-transitive graphs are the arctransitive, half-arc-transitive and zero-symmetric graphs, and their arc-types are as follows:

- Arc-transitive graphs of valency $d$ have arc-type $d$;

- Half-arc-transitive graphs of even valency $d$ have arc-type $(d / 2+d / 2)$;

- Zero-symmetric graphs have arc-type $1+\ldots+1+(1+1)+\ldots+(1+1)$.

In particular, it follows that there are $\lfloor d / 2\rfloor+1$ possibilities for the arc-type of a $d$-valent zero-symmetric graph (or GRR).

At this point, we recall that the arc-type of a VT graph $X$ depends on the action of the $G=\operatorname{Aut}(X)$ on the arcs of $X$, and in particular, the action of the vertex-stabiliser $G_{v}$ on the neighbourhood $X(v)$ of a given vertex $v$. The summands in the arc-type of $X$ are just 
the sizes of the orbits of $G_{v}$ on the neighbourhood of a vertex $v$ of $X$, while the brackets depend on the pairings of arc-orbits of $G$.

By finding the generating function for the set of marked partitions, we can count the (maximum) number of possible arc-types for each valency $d$.

Let $t^{\prime}(d, k)$ denote the number of marked partitions of $d$ with $k$ parts, and let $t(d)$ denote the number of marked partitions of an integer $d$. Obviously, $t(d)=\sum_{k} t^{\prime}(d, k)$. We can obtain the generating function $T^{\prime}(x, y)$ for $t^{\prime}(d, k)$ by adapting the generating function for standard partitions, to take account of the bracketed pairs. This can be found from [30, p. 95], for example, and is as follows:

$$
T^{\prime}(x, y)=\sum_{d \geq 0} \sum_{k \geq 0} t^{\prime}(d, k) x^{d} y^{k}=\prod_{n \geq 1} \frac{1}{\left(1-y x^{n}\right)\left(1-y^{2} x^{2 n}\right)} .
$$

Then by taking $y=1$, we get

$$
T(x)=T^{\prime}(x, 1)=\prod_{n \geq 1} \frac{1}{\left(1-x^{n}\right)\left(1-x^{2 n}\right)}=\sum_{d \geq 0} t(d) x^{d}
$$

as the generating function for $t(d)$ itself.

Here we remark that a different combinatorial approach can be taken for the generating function $T(x)$, namely through refining integer partitions by labelling some even parts (with an asterisk). For example, the partition $6=2+2+1+1$ gives rise to three labelled partitions: $6=2+2+1+1,6=2+2^{*}+1+1$, and $6=2^{*}+2^{*}+1+1$.

Now let $t^{*}(d, k)$ denote the number of labelled partitions of an integer $d$ having $k$ parts. Then the generating function for $t^{*}(d, k)$ is

$$
T^{*}(x, y)=\sum_{d \geq 0} \sum_{k \geq 0} t^{*}(d, k) x^{d} y^{k}=\prod_{n \geq 1} \frac{1}{\left(1-y x^{n}\right)\left(1-y x^{2 n}\right)},
$$

and we find that $T^{*}(x, 1)=T^{\prime}(x, 1)=T(x)$.

Also we note that the generating function $T(x)$ defines the sequence

$$
1,1,3,4,9,12,23,31,54,73,118, \ldots,
$$

which is denoted by A002513 in The On-Line Encyclopedia of Integer Sequences [24].

Finally, it should come as no surprise that marked partitions of a positive integer $d$ can be used also to count different types of solutions of a real polynomial equation of degree $d$, when attention is paid whether the roots are real and unequal, real and equal (in various combinations) or simple or multiple complex conjugate; see [4].

\section{Edge-types and arc-types for small valency}

In this section we give examples of vertex-transitive graphs with every possible edge-type and arc-type, for valencies up to 4, and summarise the information in Table 1 at the end. Note that it is enough to find examples of VT graphs for each arc-type, since the same graphs will give also all the possible edge-types. We do not give a proof that the arc-type is as claimed in each case, since that can be easily verified by computer (using for example Magma [2]), or in some cases by hand. 
Graphs with arc-type $1+1+1$ of order up to 120 are given in [8, Part III], and graphs with arc-type $(1+1)+1$ of order up to 120 are given in [8, Part II]. The other zerosymmetric graphs listed here were found with the help of Magma [2], by checking the Cayley graphs for certain kinds of generating sets for small groups. In some cases, we give the smallest possible example with the given arc-type. Some examples were found by also checking tables of vertex-transitive graphs on up to 31 vertices; see [22].

\section{Valency $d=1$ (one case):}

(P1) There is only one marked partition of 1 , namely 1 , and only one VT graph with this arc-type, namely the complete graph $K_{2}$.

\section{Valency $d=2$ (three cases):}

(P2) $2=2$ : For every $n \geq 3$, the simple cycle $C_{n}$ has arc-type 2 .

(P3) $2=1+1$ : No VT graph has arc-type $1+1$, because cycles are the only connected regular graphs with valency 2 .

(P4) $2=(1+1)$ : No VT graph has arc-type $(1+1)$, because cycles are the only connected regular graphs with valency 2 .

\section{Valency $d=3$ (four cases):}

(P5) $3=3$ : The VT graphs with arc-type 3 are precisely the arc-transitive cubic graphs, and there are infinitely many examples, the smallest of which is the complete graph $K_{4}$. Numerous other small examples, including the ubiquitous Petersen graph, are given in the Foster census [9], which was later expanded by Conder and Dobcsányi [6], and again further by Conder [5] up to order 10000.

(P6) $3=2+1$ : The smallest VT graph with arc-type $2+1$ is the triangular prism, on 6 vertices; see Figure 1. It is easy to see that the edges on the two triangles form one edge-orbit, while all the other edges form another orbit.

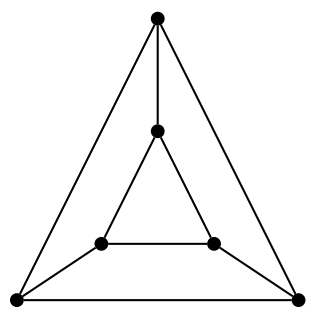

Figure 1: The triangular prism, which has arc-type $2+1$

(P7) $3=1+1+1$ : The smallest VT graph with arc-type $1+1+1$ is the zero-symmetric graph on 18 vertices from [8, p. 4]; see also Figure 2. This is the Cayley graph of a group of order 18 generated by three involutions, and it can also be described as a cubic Hamiltonian graph with LCF-code $[5,-5]^{9}$. 


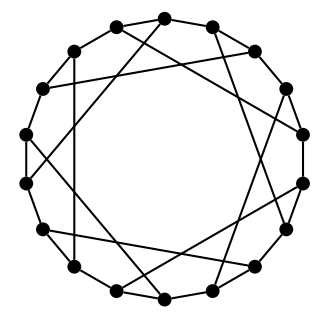

Figure 2: The smallest VT graph with arc-type $1+1+1$, on 18 vertices

(P8) $3=1+(1+1)$ : The smallest VT graph with arc-type $1+(1+1)$ is the zero-symmetric graph on 20 vertices from [8, p. 35]; see Figure 3. This is the Cayley graph of a group of order 20, generated by one involution and one non-involution, and it can also be described as a cubic Hamiltonian graph with LCF-code $[6,6,-6,-6]^{5}$. For more properties of this graph, see Lemma 8.4.

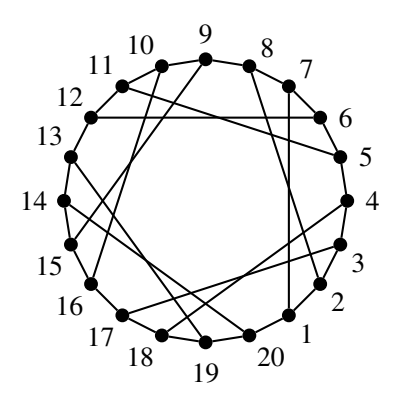

Figure 3: The smallest VT graph with arc-type $1+(1+1)$, on 20 vertices

\section{Valency $d=4$ (nine cases):}

(P9) $4=4$ : The VT graphs with arc-type 4 are arc-transitive quartic graphs. The smallest example is the complete graph $K_{5}$.

(P10) $4=(2+2)$ : The VT graphs with arc-type $(2+2)$ are half-arc-transitive quartic graphs. The smallest example is the Holt graph [12], of order 27; see Figure 4.

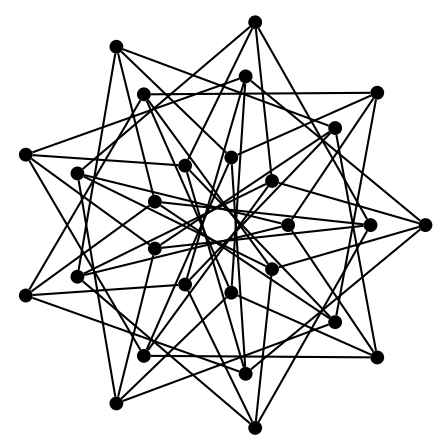

Figure 4: The Holt graph (the smallest 4-valent half-arc-transitive graph) 
(P11) $4=3+1$ : The smallest VT graph with arc-type $3+1$ is $K_{4} \square K_{2}$, which is the Cartesian product of $K_{4}$ and $K_{2}$. The two summands $K_{4}$ and $K_{2}$ have arc-types 3 and 1 , respectively, and are 'relatively prime'. The example will be generalised in Theorem 6.6.

(P12) $4=2+2$ : The smallest VT graph with arc-type $2+2$ is the circulant graph $\operatorname{Cay}\left(\mathbb{Z}_{7} ;\{1,2\}\right)$ on 7 vertices, where $\mathbb{Z}_{7}$ is viewed as an additive group. This graph is shown in Figure 5. Each of the edges on the outer 7-cycle lies in two triangles while each edge of the inner 7-cycle lies in only one triangle, and it follows that $\operatorname{Cay}\left(\mathbb{Z}_{7} ;\{1,2\}\right)$ has two edge orbits.

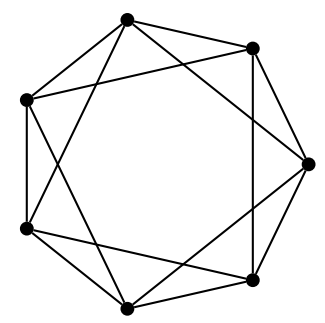

Figure 5: The circulant $\operatorname{Cay}\left(\mathbb{Z}_{7} ;\{1,2\}\right)$, which has arc-type $2+2$

(P13) $4=2+(1+1)$ : The graph on 40 vertices in Figure 6 is the smallest known VT graph with arc-type $2+(1+1)$. It is a thickened cover of the trivalent graph on 20 vertices with arc-type $(1+1)+1$; see Section 7 for generalisations of this.

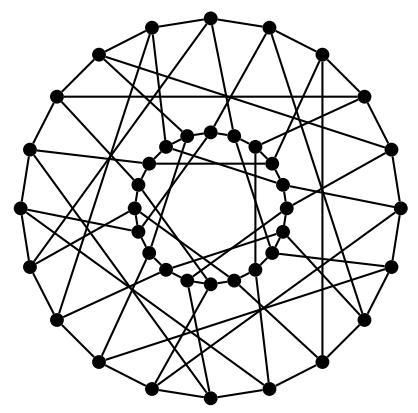

Figure 6: A VT graph with arc-type $2+(1+1)$, on 40 vertices

(P14) $4=(1+1)+(1+1)$ : The graph on 42 vertices in Figure 7 is the smallest VT graph with arc-type $(1+1)+(1+1)$. As a GRR, it is a Cayley graph of the group $C_{7} \rtimes C_{6}$ with generating set that contains an element of order 6 , an element of order 7 , and their inverses. For more details on this graph see Lemma 8.6. 

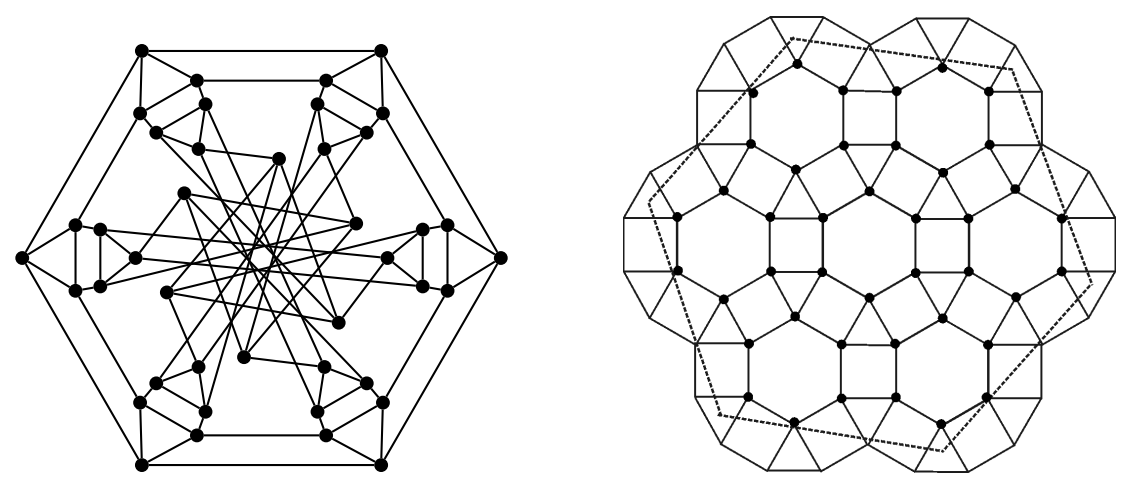

Figure 7: On the left is the smallest VT graph with arc-type $(1+1)+(1+1)$, on 42 vertices - and on the right is an illustration of an embedding of this graph on the torus, using a hexagon with opposite sides identified

(P15) $4=2+1+1$ : The graph on 12 vertices in Figure 8 is the smallest VT graph with arc-type $2+1+1$. It can be obtained from the hexagonal prism by adding diagonals to three non-adjacent quadrangles.

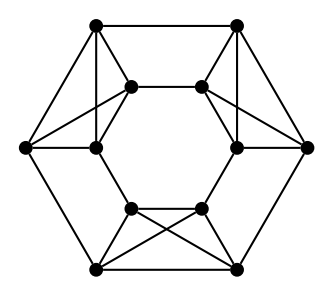

Figure 8: The smallest VT graph with arc-type $2+1+1$, on 12 vertices

(P16) $4=1+1+(1+1)$ : The graph on 20 vertices in Figure 9 is the smallest VT graph with arc-type $1+1+(1+1)$. If $G$ is the Frobenius group $C_{5} \rtimes C_{4}$ of order 20, generated by the permutations $a=(1,2,3,4,5)$ and $b=(2,3,5,4)$, which satisfy the relations $a^{5}=b^{4}=1$ and $b^{-1} a b=a^{2}$, then this graph is the Cayley graph (in fact a GRR) for $G$ given by the generating set $S=\left\{a b^{2}, a^{2} b^{2}, b, b^{-1}\right\}$.

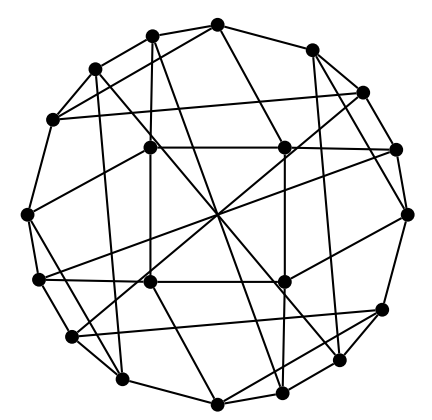

Figure 9: The smallest VT graph with arc-type $1+1+(1+1)$, on 20 vertices 
(P17) $4=1+1+1+1$ : The graph on 16 vertices in Figure 10 is the smallest VT graph with arc-type $1+1+1+1$. It is the Cayley graph (in fact a GRR) for the dihedral group $D_{8}=\left\langle x, y \mid x^{2}=y^{8}=(x y)^{2}=1\right\rangle$ of order 16 with generating set $S=\left\{x, x y, x y^{2}, x y^{4}\right\}$.

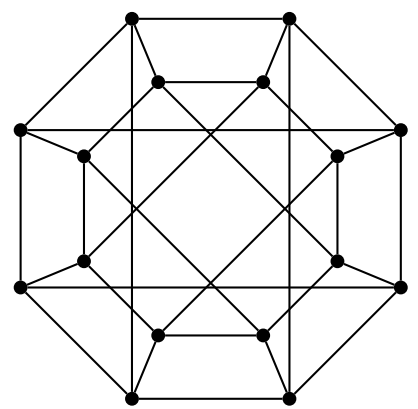

Figure 10: The smallest VT graph with arc-type $1+1+1+1$, on 16 vertices

The above examples are summarised in Table 1.

\begin{tabular}{|c|c|c|c|c|}
\hline Valency & Edge-type & Arc-type & Example & Case \\
\hline 1 & 1 & 1 & $K_{2}$ & P1 \\
\hline \multirow[t]{2}{*}{2} & \multirow[t]{2}{*}{2} & 2 & $C_{3}$ & $\mathrm{P} 2$ \\
\hline & & $(1+1)$ & [Impossible] & P3 \\
\hline 2 & $1+1$ & $1+1$ & [Impossible] & $\mathrm{P} 4$ \\
\hline 3 & 3 & 3 & $K_{4}$ & P5 \\
\hline \multirow[t]{2}{*}{3} & \multirow[t]{2}{*}{$2+1$} & $2+1$ & prisms & P6 \\
\hline & & $(1+1)+1$ & $L C F[6,6,-6,-6]^{5}$ & P7 \\
\hline 3 & $1+1+1$ & $1+1+1$ & $L C F[5,-5]^{9}$ & P8 \\
\hline \multirow[t]{2}{*}{4} & \multirow[t]{2}{*}{4} & 4 & $K_{5}$ & P9 \\
\hline & & $(2+2)$ & Holt graph & P10 \\
\hline 4 & $3+1$ & $3+1$ & $K_{4} \square K_{2}$ & P11 \\
\hline \multirow[t]{3}{*}{4} & \multirow[t]{3}{*}{$2+2$} & $2+2$ & $\operatorname{Cay}\left(\mathbb{Z}_{7} ;\{1,2\}\right)$ & P12 \\
\hline & & $2+(1+1)$ & Figure 6 & P13 \\
\hline & & $(1+1)+(1+1)$ & Figure 7 & P14 \\
\hline \multirow[t]{2}{*}{4} & \multirow[t]{2}{*}{$2+1+1$} & $2+1+1$ & Figure 8 & P15 \\
\hline & & $(1+1)+1+1$ & Figure 9 & P16 \\
\hline 4 & $1+1+1+1$ & $1+1+1+1$ & Figure 10 & P17 \\
\hline
\end{tabular}

Table 1: Edge-types and arc-types of VT graphs with valency up to 4

\section{Arc-types of Cartesian products}

Given a pair of graphs $X$ and $Y$ (which might or might not be distinct), the Cartesian product $X \square Y$ is a graph with vertex set $V(X) \times V(Y)$, such that two vertices $(u, x)$ and $(v, y)$ are adjacent in $X \square Y$ if and only if $u=v$ and $x$ is adjacent with $y$ in $Y$, or $x=y$ and $u$ is adjacent with $v$ in $X$. 
This definition can be extended to the Cartesian product $X_{1} \square \ldots \square X_{k}$ of a larger number of graphs $X_{1}, \ldots, X_{k}$. The terms $X_{i}$ are called the factors of the Cartesian product $X_{1} \square \ldots \square X_{k}$. The Cartesian product operation $\square$ is associative and commutative. A good reference for studying this and other products is the book by Imrich and Klavžar [13].

There are many properties of Cartesian product graphs that can be easily derived from the properties of their factors. For example, we have the following:

Proposition 6.1. A Cartesian product graph is connected if and only if all of its factors are connected.

Proposition 6.2. Let $X_{1}, \ldots, X_{k}$ be regular graphs with valencies $d_{1}, \ldots, d_{k}$. Then their Cartesian product $X_{1} \square \ldots \square X_{k}$ is also regular, with valency $d_{1}+\cdots+d_{k}$.

A graph $X$ is called prime (with respect to the Cartesian product) if it is not isomorphic to the Cartesian product of a pair of smaller, non-trivial graphs. It is well-known that every connected graph can be decomposed to a Cartesian product of prime graphs, which is unique up to reordering and isomorphism of the factors; for a proof, see [13, Theorem 4.9]. Similarly, two graphs are said to be relatively prime (with respect to the Cartesian product) if there is no non-trivial graph that is a factor of both. Note that two prime graphs are relatively prime unless they are isomorphic.

We are interested in the question of how the symmetries of individual graphs are involved in the symmetries of their product. Let $X=X_{1} \square \ldots \square X_{k}$, and let $\alpha$ be an automorphism of one of the $X_{i}$. Then $\alpha$ induces an automorphism $\beta$ of $X$, given by

$$
\beta:\left(v_{1}, \ldots, v_{k}\right) \mapsto\left(v_{1}, \ldots, v_{i-1}, v_{i}^{\alpha}, v_{i+1}, \ldots, v_{k}\right) .
$$

The set of all automorphisms of $X$ induced in this way forms a subgroup of $\operatorname{Aut}(X)$, and if some of the factors of $X$ are isomorphic, then $\operatorname{Aut}(X)$ contains also other automorphisms that permute these factors among themselves, but if the factors of $X$ are relatively prime, then there are no other automorphisms. Indeed we have the following:

Theorem 6.3. ([13, Corollary 4.17]) Let $X$ be the Cartesian product $X=X_{1} \square \ldots \square X_{k}$ of connected pairwise relatively prime graphs $X_{1}, \ldots, X_{k}$. Then every automorphism $\varphi$ of $X$ has the property that

$$
\varphi:\left(v_{1}, \ldots, v_{k}\right) \mapsto\left(v_{1}^{\varphi_{1}}, \ldots, v_{k}^{\varphi_{k}}\right) \quad \text { for all }\left(v_{1}, \ldots, v_{k}\right) \in V(X),
$$

where $\varphi_{i}$ is an automorphism of $X_{i}$ for $1 \leq i \leq k$.

Corollary 6.4. If $X$ be the Cartesian product $X_{1} \square \ldots \square X_{k}$ of connected pairwise relatively prime graphs $X_{1}, \ldots, X_{k}$, then $\operatorname{Aut}(X) \cong \operatorname{Aut}\left(X_{1}\right) \times \cdots \times \operatorname{Aut}\left(X_{k}\right)$.

Corollary 6.5. A Cartesian product of connected graphs is vertex-transitive if and only if every factor is vertex-transitive.

Corollary 6.5 follows directly from Theorem 6.3 when the factors are pairwise relatively prime. But it is also true in the general case — for a proof, see [13, Proposition 4.18].

We now come to the key observation we need to prove our main theorem.

Theorem 6.6. Let $X_{1}, \ldots, X_{k}$ be non-trivial connected vertex-transitive graphs, with arctypes $\tau_{1}, \ldots, \tau_{k}$. Then also $X=X_{1} \square \ldots \square X_{k}$ is a connected vertex-transitive graph, and if $X_{1}, \ldots, X_{k}$ are pairwise relatively prime, then the arc-type of $X$ is $\tau_{1}+\cdots+\tau_{k}$. 
Proof. First, the graph $X$ is connected by Proposition 6.1, and vertex-transitive by Corollary 6.5. For the second part, suppose that $X_{1}, \ldots, X_{k}$ are pairwise relatively prime. Then by Corollary 6.4, we know that $\operatorname{Aut}(X) \cong \operatorname{Aut}\left(X_{1}\right) \times \cdots \times \operatorname{Aut}\left(X_{k}\right)$. Moreover, by Theorem 6.3, the stabiliser in $\operatorname{Aut}(X)$ of a vertex $\left(v_{1}, \ldots, v_{k}\right)$ of $X$ is isomorphic to $\operatorname{Aut}\left(X_{1}\right)_{v_{1}} \times \cdots \times \operatorname{Aut}\left(X_{k}\right)_{v_{k}}$.

We will now show that two arcs incident with a given vertex $u=\left(u_{1}, \ldots, u_{k}\right)$ in $X$ are in the same orbit of $\operatorname{Aut}(X)$ if and only if the corresponding arcs are in the same orbit of $\operatorname{Aut}\left(X_{i}\right)$ for some $i$, and that two such arcs are in paired orbits of $\operatorname{Aut}(X)$ if and only if the corresponding arcs of $X_{i}$ belong to paired orbits of $\operatorname{Aut}\left(X_{i}\right)$ for some $i$. This will imply that the sizes of arc-orbits of $\operatorname{Aut}(X)$ on $X$ match the sizes of arc-orbits of the subgroups $\operatorname{Aut}\left(X_{i}\right)$ on the corresponding $X_{i}$, for $1 \leq i \leq k$, and hence that the arc-type of $X$ is just the sum of the arc-types of $X_{1}, \ldots, X_{k}$.

So suppose that $u^{\prime}=\left(u_{1}^{\prime}, \ldots, u_{k}^{\prime}\right)$ and $u^{\prime \prime}=\left(u_{1}^{\prime \prime}, \ldots, u_{k}^{\prime \prime}\right)$ are adjacent to $u=$ $\left(u_{1}, \ldots, u_{k}\right)$ in $X$, and that the $\operatorname{arcs}\left(u, u^{\prime}\right)$ and $\left(u, u^{\prime \prime}\right)$ lie in the same orbit of $\operatorname{Aut}(X)$. Then there exists an automorphism $\varphi$ of $X$ taking $\left(u, u^{\prime}\right)$ to $\left(u, u^{\prime \prime}\right)$, and since $\varphi$ stabilises $u$, we know that $\varphi=\left(\varphi_{1}, \ldots, \varphi_{k}\right)$ where $\varphi_{i} \in \operatorname{Aut}\left(X_{i}\right)_{u_{i}}$ for $1 \leq i \leq k$. Also $u^{\prime}$ differs from $u$ in only one coordinate, say the $i$-th one, in which case $u_{j}^{\prime}=u_{j}$ for $j \neq i$, and then since $\varphi=\left(\varphi_{1}, \ldots, \varphi_{k}\right)$ takes $u^{\prime}$ to $u^{\prime \prime}$ (and $\varphi_{j}$ fixes $u_{j}$ ), we find that $u_{j}^{\prime \prime}=u_{j}$, so that $u^{\prime \prime}$ differs from $u$ only in the $i$-th coordinate as well. In particular, $\varphi_{i}$ fixes $u_{i}$ and takes $u_{i}^{\prime}$ to $u_{i}^{\prime \prime}$, so the $\operatorname{arcs}\left(u_{i}, u_{i}^{\prime}\right)$ and $\left(u_{i}, u_{i}^{\prime \prime}\right)$ lie in the same orbit of $\operatorname{Aut}\left(X_{i}\right)$.

The converse is easy. For suppose the $\operatorname{arcs}\left(u_{i}, u_{i}^{\prime}\right)$ and $\left(u_{i}, u_{i}^{\prime \prime}\right)$ lie in the same orbit of $\operatorname{Aut}\left(X_{i}\right)$, and $\varphi_{i}$ is an automorphism of $X_{i}$ taking $\left(u_{i}, u_{i}^{\prime}\right)$ to $\left(u_{i}, u_{i}^{\prime \prime}\right)$. Then letting $u_{j}^{\prime}=u_{j}^{\prime \prime}=u_{j}$ for $j \neq i$, and $u^{\prime}=\left(u_{1}^{\prime}, \ldots, u_{k}^{\prime}\right)$ and $u^{\prime \prime}=\left(u_{1}^{\prime \prime}, \ldots, u_{k}^{\prime \prime}\right)$, we find that the automorphism of $X$ induced by $\varphi_{i}$ takes $\left(u, u^{\prime}\right)$ to $\left(u, u^{\prime \prime}\right)$, and so these two arcs lie in the same orbit of $\operatorname{Aut}(X)$.

On the other hand, suppose the arcs $\left(u, u^{\prime}\right)$ and $\left(u, u^{\prime \prime}\right)$ lie in different but paired orbits of $\operatorname{Aut}(X)$. Then there exists an automorphism $\varphi$ of $X$ taking $\left(u, u^{\prime}\right)$ to $\left(u^{\prime \prime}, u\right)$, and $\varphi=\left(\varphi_{1}, \ldots, \varphi_{k}\right)$ where $\varphi_{i} \in \operatorname{Aut}\left(X_{i}\right)$ for $1 \leq i \leq k$. Again, $u^{\prime}$ differs from $u$ in only one coordinate, say the $i$-th one, in which case $u_{j}^{\prime}=u_{j}$ for $j \neq i$. Then since $\varphi$ takes $u^{\prime}$ to $u$, we find that $\varphi_{j}$ fixes $u_{j}$, and since $\varphi$ takes $u$ to $u^{\prime \prime}$, also $u_{j}^{\prime \prime}=u_{j}$. Thus, as before, $u^{\prime \prime}$ differs from $u$ only in the $i$-th coordinate, and the arcs $\left(u_{i}, u_{i}^{\prime}\right)$ and $\left(u_{i}, u_{i}^{\prime \prime}\right)$ lie in the same orbit of $\operatorname{Aut}\left(X_{i}\right)$. The converse is analogous to the previous case, and this completes the proof.

Our final observations in this section are often helpful when proving that a given graph is prime with respect to the Cartesian product. The first two are easy, and the third one is proved in [14], for example.

Lemma 6.7. Let $X=X_{1} \square X_{2}$ be a Cartesian product of two connected graphs, each of which has at least two vertices. Then every edge of $X$ is contained in a 4-cycle.

Corollary 6.8. Let $X$ be a connected graph. If some edge of $X$ is not contained in any 4-cycle, then $X$ is prime. In particular, if $X$ has no 4-cycles, then $X$ is prime.

Lemma 6.9. Let $X$ be a Cartesian product of connected graphs.

(a) All the edges in a cycle of length 3 in $X$ belong to the same factor of $X$.

(b) Let $(v, u, w, x)$ be any 4-cycle in $X$. Then the edges $\{v, u\}$ and $\{w, x\}$ belong to the same factor of $X$, as do the edges $\{u, w\}$ and $\{x, v\}$. 
(c) If e and $f$ are incident edges that are not in the same factor of $X$, then there exists $a$ unique 4-cycle that contains $e$ and $f$, and this 4-cycle has no diagonals.

\section{Thickened covers}

In this section we explain the general notion of a thickened cover of a graph, and show how it can be used to build larger vertex-transitive graphs from a given one.

Let $X$ be any simple graph, $F$ any subset of the edge-set of $X$, and $m$ any positive integer. Then we define $X(F, m)$ to be the graph with vertex set $V(X) \times \mathbb{Z}_{m}$, and with edges of two types:

(a) an edge from $(u, i)$ to $(v, i)$, for every $i \in \mathbb{Z}_{m}$ and every $\{u, v\} \in E(X) \backslash F$,

(b) an edge from $(u, i)$ to $(v, j)$, for every $(i, j) \in \mathbb{Z}_{m} \times \mathbb{Z}_{m}$ and every $\{u, v\} \in F$.

We call $X(F, m)$ a thickened $m$-cover of $X$ over $F$.

In other words, a thickened $m$-cover of a graph $X$ over a given set $F$ of edges of $X$ is obtained by replacing each vertex of $X$ by $m$ vertices, and each edge by the complete bipartite graph $K_{m, m}$ if the edge lies in $F$, or by $m K_{2}$ (a set of $m$ 'parallel' edges) if the edge does not lie in $F$.

For example, the thickened 3 -cover of the path graph $P_{6}$ on 6 vertices over the unique 1-factor of $P_{6}$ is shown in Figure 11.

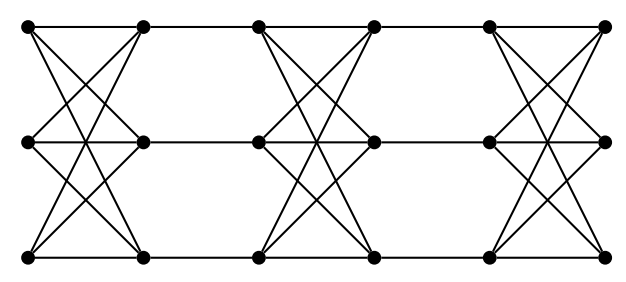

Figure 11: A thickened 3-cover of $P_{6}$ (over its 1-factor)

Here we note that $X(F, 1)$ is isomorphic to $X$, while $X(\emptyset, m)$ is isomorphic to $m X$ (the union of $m$ copies of $X$ ).

Also the base graph $X$ is a quotient of $X(F, m)$, obtainable by identifying all vertices $(u, i)$ that have the same first coordinate, but $X(F, m)$ is not a covering graph of $X$ in the usual sense of that term when $F$ is non-empty and $m>1$, because in that case the valency of a vertex $(u, i)$ of $X(F, m)$ is greater than the valency of $u$.

On the other hand, if $X^{(m)}$ is the multigraph obtained from $X$ by replacing each edge from $F$ by $m$ parallel edges, then $X(F, m)$ is a regular covering graph of $X^{(m)}$, with voltages taken from $\mathbb{Z}_{m}$ : we may choose the voltages of the edges not in $F$ to be 0 , and for each set of $m$ parallel edges, choose a direction and then assign distinct voltages from $\mathbb{Z}_{m}$ to these edges. The derived graph of $X^{(m)}$ with this voltage assignment is a covering graph of $X^{(m)}$ that is isomorphic to $X(F, m)$.

(For the definitions of voltage graphs and covering graphs, see the book on topological graph theory by Gross and Tucker [10].)

For each $u \in V(X)$, we may call the set $\left\{(u, i): i \in \mathbb{Z}_{m}\right\}$ of vertices of $X(F, m)$ the fibre over the vertex $u$ of $X$. Similarly the fibre over the edge $\{u, v\}$ of $X$ is the set $\left\{\{(u, i),(v, i)\}: i \in \mathbb{Z}_{m}\right\}$ of edges of $X(F, m)$ if $\{u, v\} \in E(X) \backslash F$, or the set 
$\left\{\{(u, i),(v, j)\}: i, j \in \mathbb{Z}_{m}\right\}$ if $\{u, v\} \in F$. Also for each $i \in \mathbb{Z}_{m}$ we call the subgraph of $X(F, m)$ induced by the vertices $\{(u, i): u \in V(X)\}$ the $i$-th layer of $X(F, m)$.

We now define three families of bijections on the vertex set of $X(F, m)$. The first is $\tilde{\varphi}$, which is induced by addition of $1 \bmod m$ on $\mathbb{Z}_{m}$, and given by the rule

$$
\tilde{\varphi}:(u, i) \mapsto(u, i+1) \quad \text { for all } u \in V(X) \text { and all } i \in \mathbb{Z}_{m} .
$$

Next, if $\psi$ is any automorphism of $X$, then we define $\tilde{\psi}$ by the rule

$$
\tilde{\psi}:(u, i) \mapsto\left(u^{\psi}, i\right) \quad \text { for all } u \in V(X) \text { and all } i \in \mathbb{Z}_{m} .
$$

Finally, if $i, j \in \mathbb{Z}_{m}$ and $\{u, v\}$ is an edge in $F$ such that $u$ and $v$ lie in different components of $X \backslash F$ (the graph obtained from $X$ by deleting all the edges in $F$ ), then we define the bijection $\tilde{\theta}=\tilde{\theta}(u, v, i, j)$ by

$$
\tilde{\theta}:(w, k) \mapsto \begin{cases}(w, j) & \text { if } k=i \text { and } w \text { lies in the same component of } X \backslash F \text { as } v, \\ (w, i) & \text { if } k=j \text { and } w \text { lies in the same component of } X \backslash F \text { as } v, \\ (w, k) & \text { otherwise. }\end{cases}
$$

Lemma 7.1. If $X$ is any graph, $F \subseteq E(X)$ and $m \geq 2$, then $\tilde{\varphi}$ is an automorphism of $X(F, m)$.

Proof. The mapping $\tilde{\varphi}$ is a bijection and obviously sends edges of $X(F, m)$ to edges, so is an automorphism of $X(F, m)$.

Lemma 7.2. If $\psi \in \operatorname{Aut}(X)$ and $\psi$ preserves $F$ setwise, then $\tilde{\psi}$ is an automorphism of $X(F, m)$ for all $m$.

Proof. The given mapping $\tilde{\psi}$ is clearly a bijection. Next, if $\{u, v\} \in F$ then also $\left\{u^{\psi}, v^{\psi}\right\} \in$ $F$ by hypothesis, and so $\{(u, i),(v, j)\}^{\tilde{\psi}}=\left\{\left(u^{\psi}, i\right),\left(v^{\psi}, j\right)\right\}$ is an edge of $X(F, m)$, for all $i, j \in \mathbb{Z}_{m}$. Similarly, if $\{u, v\} \in E(X) \backslash F$, then $\left\{u^{\psi}, v^{\psi}\right\} \in E(X) \backslash F$, and so $\{(u, i),(v, i)\}^{\tilde{\psi}}=\left\{\left(u^{\psi}, i\right),\left(v^{\psi}, i\right)\right\}$ is an edge of $X(F, m)$, for all $i \in \mathbb{Z}_{m}$.

Corollary 7.3. If $X$ is a vertex-transitive graph, and $F \subseteq E(X)$ is a union of some edgeorbits of $X$, then $X(F, m)$ is vertex-transitive for every $m \geq 2$.

Proof. The subgroup of $\operatorname{Aut}(X(F, m))$ generated by $\tilde{\varphi}$ and $\{\tilde{\psi}: \psi \in \operatorname{Aut}(X)\}$ acts transitively on the vertex set of $X(F, m)$.

Here we note that $X(F, m)$ is vertex-transitive also when $F$ is the union of edge-orbits of some vertex-transitive subgroup of $\operatorname{Aut}(X)$.

Lemma 7.4. If $X$ is any graph, $F \subseteq E(X)$ and $m \geq 2$, and $\{u, v\}$ is any edge in $F$ such that $u$ and $v$ lie in different components of $X \backslash F$, then $\tilde{\theta}=\tilde{\theta}(u, v, i, j)$ is an automorphism of $X(F, m)$, for all $i, j \in \mathbb{Z}_{m}$.

Proof. The mapping $\tilde{\theta}$ is clearly a bijection, and to prove it is an automorphism of $X(F, m)$, all we have to do is show that it preserves the set $E^{\prime}$ of edges incident with one or more vertices not fixed by $\tilde{\theta}$. So suppose $w$ is any vertex of $X$ lying in the same component of $X \backslash F$ as $v$, and consider the effect of $\tilde{\theta}$ on an edge from (say) the vertex $(w, i)$ to a vertex 
$(z, k)$ in $X(F, m)$. If $\{w, z\} \in E(X) \backslash F$ and $k=i$, then $z$ lies in the same component of $X \backslash F$ as $w$ and hence in the same one as $v$, and therefore $\tilde{\theta}$ takes $(w, i)$ to $(w, j)$, and $(z, k)=(z, i)$ to $(z, j)$, which is a neighbour of $(w, j)$. On the other hand, if $\{w, z\} \in F$ and $k$ is arbitrary, then $\tilde{\theta}$ takes $(w, i)$ to $(w, j)$, and $(z, k)$ to $(z, k)$, which is a neighbour of $(w, j)$. The analogous things happen for edges incident with $(w, j)$ in place of $(w, i)$, and so the set $E^{\prime}$ is preserved by $\tilde{\theta}$, as required.

Next, we note that under the assumptions of Lemma 7.4, the automorphism $\tilde{\theta}(u, v, i, j)$ fixes the vertex $(u, k)$, for every $k \in \mathbb{Z}_{m}$, and therefore the stabiliser of every such $(u, k)$ contains the automorphisms $\tilde{\theta}(u, v, i, j)$ for all $i, j \in \mathbb{Z}_{m}$.

We now give a helpful example of an application of this thickened cover construction, to cycles of even order.

Theorem 7.5. Let $X$ be the cycle on $n$ vertices, where $n$ is even and $n>2$, and let $F$ be a 1-factor of $X$. Then $X(F, m)$ is vertex-transitive for all $m \geq 2$, with arc-type $m+1$ (that is, with two self-paired arc orbits of lengths $m$ and 1$)$ whenever $(n, m) \neq(4,2)$. Also $X(F, m)$ is prime with respect to the Cartesian product, for $m \geq 2$ and $n \neq 4$.

Proof. We may take $V(X)=\mathbb{Z}_{n}$ and $E(X)=\left\{\{r, r+1\}: r \in \mathbb{Z}_{n}\right\}$, and assume without loss of generality that $F=\left\{\{2 r, 2 r+1\}: r \in \mathbb{Z}_{n}\right\}$. By Lemma 7.1 and Lemma 7.4, we know that $\tilde{\varphi}$ and $\tilde{\theta}(2 r, 2 r+1, i, j)$ are automorphisms of $X(F, m)$, for all $r \in \mathbb{Z}_{n}$ and all $i, j \in \mathbb{Z}_{m}$. Also let $\tilde{\rho}$ and $\tilde{\tau}$ be the permutations of $V(X(F, m))$ given by

$$
\tilde{\rho}:(u, i) \mapsto(u+2, i) \text { and } \tilde{\tau}:(u, i) \mapsto(1-u, i) \quad \text { for all } u \in V(X) \text { and all } i \in \mathbb{Z}_{m} .
$$

By Lemma 7.2, these are automorphisms of $X(F, m)$, induced by the automorphisms $\rho$ and $\tau$ of $X$ taking $u \mapsto u+2$ and $u \mapsto 1-u$, and from this is is clear that $\tilde{\rho}$ and $\tilde{\tau}$ generate a dihedral subgroup of $\operatorname{Aut}(X(F, m))$ of order $n$ (with $n / 2$ 'rotations' and $n / 2$ 'reflections'). Moreover, this subgroup acts transitively on the vertices of the $i$-th layer $\left\{(u, i): u \in \mathbb{Z}_{n}\right\}$ of $X(F, m)$, for every $i \in \mathbb{Z}_{m}$. It follows that the subgroup of $\operatorname{Aut}(X(F, m))$ generated by the automorphisms $\tilde{\varphi}, \tilde{\rho}$ and $\tilde{\tau}$ acts transitively on the set of all vertices of $X(F, m)$, and therefore $X(F, m)$ is vertex-transitive.

Now let $\Delta_{1}$ and $\Delta_{2}$ be the sets of arcs associated with edges of the types (a) and (b) from the construction of $X(F, m)$. Specifically, let $\Delta_{1}$ be the set of arcs associated with edges of the form $\{(2 r+1, i),(2 r+2, i)\}$ for $r \in \mathbb{Z}_{n}$ and $i \in \mathbb{Z}_{m}$, and let $\Delta_{2}$ be the set of arcs associated with edges of the form $\{(2 r, i),(2 r+1, j)\}$ for $r \in \mathbb{Z}_{n}$ and $i, j \in \mathbb{Z}_{m}$. Note that by the thickening construction, every vertex of $X(F, m)$ is incident with one arc from $\Delta_{1}$, and with $m$ arcs from $\Delta_{2}$.

All the arcs in $\Delta_{1}$ lie in the same orbit of $\operatorname{Aut}(X(F, m))$. In fact $\Delta_{1}$ is an arc-orbit of the subgroup generated by $\tilde{\varphi}, \tilde{\rho}$ and $\tilde{\tau}$, and here we may note that $\tilde{\tau} \tilde{\rho}$ reverses each arc $((1, i),(2, i))$, and so $\tilde{\rho}^{-r}(\tilde{\tau} \tilde{\rho}) \tilde{\rho}^{r}$ reverses each arc $((2 r+1, i),(2 r+2, i))$ in $\Delta_{1}$. Similarly, all the arcs associated with edges of the form $\{(2 r, i),(2 r+1, i)\}$ for $r \in \mathbb{Z}_{n}$ and $i \in \mathbb{Z}_{m}$ lie in the same orbit of the subgroup generated by $\tilde{\varphi}, \tilde{\rho}$ and $\tilde{\tau}$, and then since $\tilde{\theta}(2 r, 2 r+1, i, j)$ interchanges the vertices $(2 r, i)$ and $(2 r, j)$ while fixing $(2 r+1, i)$ and $(2 r+1, j)$, we find that all the arcs in $\Delta_{2}$ lie in the same orbit of $\operatorname{Aut}(X(F, m))$.

Next, we show there is no automorphism taking an arc in $\Delta_{1}$ to an arc in $\Delta_{2}$, unless $(n, m)=(4,2)$. To see this, we consider the number of quadrangles containing a given edge. Every edge of the form $\{(2 r, i),(2 r+1, j)\}$ is contained in at least $(m-1)^{2}$ quadrangles, namely those with vertices $(2 r, i),(2 r+1, j),(2 r, k)$ and $(2 r+1, \ell)$, for given 
$k \in \mathbb{Z}_{m} \backslash\{i\}$ and $\ell \in \mathbb{Z}_{m} \backslash\{j\}$. On the other hand, if $n>4$ then no edge of the form $\{(2 r+1, i),(2 r+2, i)\}$ is contained in a quadrangle, because the other neighbours of $(2 r+1, i)$ and $2 r+2, i)$ are all of the form $(2 r, j)$ and $(2 r+3, j)$ respectively, and no two of these are adjacent, while if $n=4$, then every edge of the form $\{(2 r+1, i),(2 r+2, i)\}$ is contained in exactly $m$ quadrangles, namely those with vertices $(2 r+1, i),(2 r+2, i)$, $(2 r+3, j)$ and $(2 r, j)$, for given $j \in \mathbb{Z}_{m}$. Since $(m-1)^{2}>m$ for all $m>2$, the numbers of quadrangles are different when $(n, m) \neq(4,2)$.

Hence if $(n, m) \neq(4,2)$, we find that $\Delta_{1}$ and $\Delta_{2}$ are arc-orbits of $X(F, m)$. Then since every vertex is incident with one arc from $\Delta_{1}$ and $m$ arcs from $\Delta_{2}$, the arc-type of $X(F, m)$ is $m+1$ in this case. Finally, if $n>4$, then by Corollary 6.8, the fact that not every edge of $X(F, m)$ is contained in a quadrangle implies that $X(F, m)$ is prime with respect to the Cartesian product.

In the exceptional case $(n, m)=(4,2)$, the graph $C_{4}(F, 2)$ is isomorphic to the 3-cube $Q_{3}$, which is arc-transitive (with arc-type 3 ). Also for every $m \geq 2$, the graph $C_{4}(F, m)$ is isomorphic to the Cartesian product of $K_{m, m}$ and $K_{2}$, and hence is not prime.

Thickened covers of cycles belong to the family of cyclic Haar graphs [11], which are regular covering graphs over a dipole. Indeed the graph $C_{2 k}(F, m)$ we considered in Theorem 7.5 is a covering graph over a dipole with $n+1$ edges, and voltage assignments $1,0, m, 2 m, \ldots,(n-1) m$ from the additive group $\mathbb{Z}_{m n}$.

Next, we prove the following, which will be very helpful later.

Theorem 7.6. Let $X$ be a vertex-transitive graph, let $F$ be union of edge-orbits of $X$, and let $m$ be any integer with $m \geq 2$. Also suppose that for every edge $\{u, v\} \in F$, the vertices $u$ and $v$ lie in different components of $X \backslash F$, and let $(x, y)$ and $(z, w)$ be two arcs lying in the same arc-orbit of $X$. Then

(a) the arcs $((x, i),(y, i))$ and $((z, j),(w, j))$ lie in the same arc-orbit of $X(F, m)$ for all $i, j, \in \mathbb{Z}_{m}$, and

(b) the arcs $((x, i),(y, j))$ and $((z, k),(w, \ell))$ lie in the same arc-orbit of $X(F, m)$ for all $i, j, k, \ell \in \mathbb{Z}_{m}$, when $\{x, y\} \in F$.

Proof. First, there exists an automorphism $\psi$ that maps $(x, y)$ to $(z, w)$. Now let $\tilde{\varphi}$ and $\tilde{\psi}$ be the mappings defined earlier in this section in (7.1) and (7.2). These are automorphisms, by Lemma 7.1 and Lemma 7.2 , and $\tilde{\varphi}^{j-i} \tilde{\psi}$ takes the arc $((x, i),(y, i))$ to $((z, j),(w, j))$, and so these two arcs lie in the same orbit of $\operatorname{Aut}(X(F, m))$.

Next, suppose $\{x, y\} \in F$. Then also $\{z, w\} \in F$, since $\{x, y\}$ and $\{z, w\}$ are in the same edge-orbit, and the vertices $z$ and $w$ lie in different components of $X \backslash F$, by hypothesis. Note that the automorphism $\tilde{\varphi}^{k-i} \tilde{\psi}$ takes $((x, i),(y, i))$ to $((z, k),(w, k))$, Now let $\tilde{\theta}=\tilde{\theta}(z, w, k, \ell)$, as defined in (7.3). This is an automorphism of $X(F, m)$, by Lemma 7.4 , which takes $((z, k),(w, k))$ to $((z, k),(w, \ell))$. Thus $\tilde{\varphi}^{k-i} \tilde{\psi} \tilde{\theta}$ takes $((x, i),(y, i))$ to $((z, k),(w, \ell))$, and so these two arcs lie in the same of $\operatorname{Aut}(X(F, m))$.

Note that Theorem 7.6 cannot be pushed much further. For example, if $F$ is a 1-factor in $X=C_{6}$, then the graph $Y=C_{6}(F, 2)$ has arc-type $2+1$, by Theorem 7.5. Now one might expect that if $\Phi_{1}$ is the smaller edge-orbit of $Y$, then the 4-valent graph $Y\left(\Phi_{1}, 2\right)$ has arc-type $2+2$, but this does not happen: it turns out that $Y\left(\Phi_{1}, 2\right)$ is arc-transitive, and so has arc-type 4 . 


\section{Building blocks}

In this section we produce families of examples (and a few single examples) of vertextransitive graphs with certain arc-types, which we will use as building blocks for the Cartesian product construction, to prove our main theorem in the final section. The marked partitions that occur as arc-types in these cases have a small number of summands. We begin with the arc-transitive case, for which there is just one summand.

Lemma 8.1. For every integer $m \geq 2$, there exist infinitely many VT graphs that have arc-type $m$ and are prime with respect to the Cartesian product.

Proof. First, when $m=2$ we can take the cycle graphs $C_{n}$, for $n \geq 5$. These are vertextransitive, with arc-type 2 , and taking $n>4$ ensures that $C_{n}$ contains no 4-cycles and is therefore prime, by Lemma 6.7.

Now suppose $m \geq 3$. We construct infinitely many $m$-valent arc-transitive graphs, using a theorem of Macbeath [16] which gives the following: for almost all positive integer triples $\left(m_{1}, m_{2}, m_{3}\right)$ with $1 / m_{1}+1 / m_{2}+1 / m_{3}<1$, there exist infinitely many odd primes $p$ for which the simple group $\operatorname{PSL}(2, p)$ is generated by two elements $x$ and $y$ such that $x, y$ and $x y$ have orders $m_{1}, m_{2}$ and $m_{3}$, respectively.

Here we can take $\left(m_{1}, m_{2}, m_{3}\right)=(2, m, m+4)$, and then for each such prime $p>m$, take $G=\operatorname{PSL}(2, p)$ and let $H$ be the cyclic subgroup of $G$ generated by $y$. Then $|H|=m$, and the double coset graph $\Gamma=\Gamma(G, H, x)$ is an arc-transitive graph of order $|G| / m=$ $p(p-1)(p+1) /(2 m)$. This graph has valency $m$, because the stabiliser in $G$ of the arc $(H, H x)$ is the cyclic subgroup $H \cap x^{-1} H x$, which is trivial since $G$ is simple. Thus $\Gamma$ has arc-type $m$.

It remains to show that $\Gamma$ is prime. For the moment, suppose that $\Gamma \cong X \square Y$ where $X$ and $Y$ are relatively prime non-trivial graphs. Then by Corollary 6.5, $X$ and $Y$ are vertex-transitive, and so by Theorem 6.6 we have $m=\operatorname{at}(\Gamma)=\operatorname{at}(X)+\operatorname{at}(Y)$, which is impossible. Hence the prime factors of $\Gamma$ must be all the same, and so $\Gamma$ is the Cartesian product of (say) $k$ copies of a single prime graph $X$. But then the order of $\Gamma$ is $|V(X)|^{k}$, which is impossible unless $k=1$, since the prime $p$ divides $|V(\Gamma)|=p(p-1)(p+1) /(2 m)$ but $p^{2}$ does not. Hence $\Gamma$ itself is prime.

At this point we remark that there are several other ways to produce infinitely many $m$-valent arc-transitive graphs for all $m \geq 3$. For example, another construction uses homological covers: start with a given $m$-valent arc-transitive graph $X$ (such as the complete graph on $m+1$ vertices), and then for every sufficiently large prime $p$, construct a homological $p$-cover $\Gamma_{p}$ over $X$ with no 4 -cycles. Then $\Gamma_{p}$ is also an $m$-valent arc-transitive graph, and is prime since it contains no 4-cycles; see [17].

Next, we consider half-arc-transitive graphs.

Lemma 8.2. For every integer $m \geq 2$, there exist infinitely many VT graphs that have arc-type $(m+m)$ and are prime with respect to the Cartesian product.

Proof. In 1970, Bouwer [3] constructed an infinite family of vertex- and edge-transitive graphs of even valency, indexed by triples $(m, k, n)$ of integers such that $m, k, n \geq 2$ and $2^{k} \equiv 1 \bmod n$. Each such graph, which we will call $B(m, k, n)$, has order $k n^{m-1}$ and valency $2 m$. The construction is easy, using only modular arithmetic. Bouwer proved that the graphs $B(m, 6,9)$ are half-arc-transitive, thereby showing that for every even integer 
$2 m>2$, there exists a half-arc-transitive graph with valency $2 m$. Recently the first and third authors of this paper adapted Bouwer's approach to prove that almost all the graphs $B(m, k, n)$ are half-arc-transitive [7]. In particular, they showed that if $n>7$ and $k>6$ (and $\left.2^{k} \equiv 1 \bmod n\right)$, then $X(m, k, n)$ is a half-arc-transitive graph of girth 6 , for every $m \geq 2$. This gives infinitely many prime graphs of type $(m+m)$, for every $m \geq 2$.

Here we note that there are several constructions for half-arc transitive graphs. In particular, Li and Sim used properties of projective special linear groups to construct infinitely many half-arc transitive graphs of every even valency greater than 2 in [15]. A census of 4-valent half-arc transitive graphs up to 1000 vertices is given in [20].

Lemma 8.3. For every integer $m \geq 2$ there exist infinitely many prime VT graphs with arc-type $m+1$.

Proof. By Theorem 7.5, for every $m \geq 2$ and every even $n>4$, the thickened $m$-cover of $C_{n}$ over a 1 -factor $F$ (of $C_{n}$ ) is a prime VT graph with arc-type $m+1$.

In fact we will need only one prime VT graph with arc-type $m+1$ for each $m$ in the proof of Theorem 9.1, as we do for the next two arc-types, $m+(1+1)$ and $1+(m+m)$, as well.

Lemma 8.4. For every integer $m \geq 2$ there exists a prime VT graph with arc-type $m+$ $(1+1)$.

Proof. Let $X$ be the graph with arc-type $1+(1+1)$ given in Figure 3. Before proceeding, we describe some additional properties of $X$. First, $\operatorname{Aut}(X)$ is generated by the involutory automorphism $\alpha$ that takes $v \mapsto 21-v$ for all $v \in V(X)$, and the automorphism $\beta$ of order 4 that acts as $(1,7,8,2)(3,20,6,9)(4,14,5,15)(10,17,19,12)(11,16,18,13)$ on vertices. In fact $\operatorname{Aut}(X)$ is isomorphic to the semi-direct product $C_{5} \rtimes_{3} C_{4}$, with normal subgroup of order 5 generated by $\gamma=\alpha \beta^{2}(=[\beta, \alpha])$, and $\beta^{-1} \gamma \beta=\gamma^{3}$. In particular, $X$ is a Cayley graph (and a GRR) for this group.

The graph $X$ has two edge-orbits: one of size 20 containing the edges $\{1,2\}$ and $\{1,7\}$, and one of size 10 containing the edge $\{1,20\}$. Edges in the first orbit lie in quadrangles, while those in the second do not. The arc $(1,20)$ is reversed by the automorphism $\alpha$, so it lies in a self-paired arc-orbit, of size 20 . On the other hand, the arcs $(1,2)$ and $(1,7)$ lie in distinct paired arc-orbits, also of size 20. (This can be seen by either considering the images of $(1,20)$ under the 20 automorphisms $\beta^{i} \gamma^{j}$ (for $i \in \mathbb{Z}_{4}$ and $j \in \mathbb{Z}_{5}$ ), or by using the effect on 7 -cycles to show there is no automorphism that takes $(1,2)$ to $(1,7)$.)

Now let $F$ be the smaller edge-orbit, containing the edges of the form $\{2 i, 2 i+1\}$ (with the vertices considered mod 20, so we treat 20 as 0 ), and let $Y$ be the thickened $m$-cover $X(F, m)$ of $X$ over $F$. Then $Y$ is vertex-transitive, by Corollary 7.3, and its valency is $m+2$.

The edges from $(1,0)$ to $(2,0)$ and $(1,0)$ to $(7,0)$ both lie in a single quadrangle, namely the one with vertices $(1,0),(2,0),(8,0)$ and $(7,0)$, while the edge from $(1,0)$ to $(20,0)$ lies in exactly $(m-1)^{2}$ quadrangles, namely the ones with third and fourth vertices $(1, i)$ and $(20, j)$ for any $i, j \in \mathbb{Z}_{m} \backslash\{0\}$. Hence if $m>2$, then the edges from $(1,0)$ to $(2,0)$ and $(1,0)$ to $(7,0)$ cannot lie in the same edge-orbit as the edge from $(1,0)$ to $(20,0)$. This is also true when $m=2$, because (for example) the edges from $(1,0)$ to $(2,0)$ and $(1,0)$ to $(7,0)$ both lie in 16 different 7 -cycles, while the edge from $(1,0)$ to $(20,0)$ lies 
in only 12 different 7 -cycles. (This can be checked by hand or by use of MAGMA.) Also $X \backslash F$ is a disjoint union of quadrangles (on vertex-sets $\{4 i+1,4 i+2,4 i+7,4 i+8\}$ for $i \in \mathbb{Z}_{5}$ ), and so Theorem 7.6 applies. By part (b) of Theorem 7.6, the edge-orbit $F$ of $X$ gives rise to a summand $m$ for the arc-type of $Y$, and then by part (a), noting that $(1,2)$ and $(7,1)$ lie in the same arc-orbit of $X$, we find that $Y=X(F, m)$ has arc-type $m+(1+1)$ or $m+2$.

To show that $Y$ has arc-type $m+(1+1)$, again we consider 7 -cycles. It is an easy exercise to show that there are exactly $4 \mathrm{~m}^{2}$ cycles of length 7 containing the edge from $(1,0)$ to $(2,0)$, namely those of the following forms:

- $((1,0),(2,0),(3, i),(4, i),(5, j),(6, j),(7,0))$, for any $i, j \in \mathbb{Z}_{m}$,

- $((1,0),(2,0),(3, i),(4, i),(18, i),(19, j),(20, j))$, for any $i, j \in \mathbb{Z}_{m}$,

- $((1,0),(2,0),(3, i),(17, i),(18, i),(19, j),(20, j))$, for any $i, j \in \mathbb{Z}_{m}$,

- $((1,0),(2,0),(8,0),(9, i),(15, i),(14, j),(20, j))$, for any $i, j \in \mathbb{Z}_{m}$.

Note that some of these can differ in only one vertex, namely in the 4th vertex of the second and third forms, for a given pair $(i, j)$. Similarly, there are exactly $4 m^{2}$ cycles of length 7 containing the edge from $(1,0)$ to $(7,0)$, namely those of the following forms:

- $((1,0),(7,0),(6, i),(12, i),(13, j),(14, j),(20, j))$, for any $i, j \in \mathbb{Z}_{m}$,

- $((1,0),(7,0),(6, i),(12, i),(13, j),(19, j),(20, j))$, for any $i, j \in \mathbb{Z}_{m}$,

- $((1,0),(7,0),(6, i),(5, i),(4, j),(3, j),(2,0))$, for any $i, j \in \mathbb{Z}_{m}$,

- $((1,0),(7,0),(8,0),(9, i),(15, i),(14, j),(20, j))$, for any $i, j \in \mathbb{Z}_{m}$.

But in these cases, when two such 7-cycles differ in only one vertex, they differ in the 6 th vertex (of the first and second form, for a given pair $(i, j)$ ). It follows that there can be no automorphism of $Y$ taking the arc $((1,0),(2,0))$ to the arc $((1,0),(7,0))$, and so the arc-type of $Y$ must be $m+(1+1)$.

It remains to show that $Y=X(F, m)$ is prime. For this, we consider any decomposition of $Y$ into Cartesian factors, which are connected by Proposition 6.1, and we apply Lemma 6.9. The edge $\{(1,0),(2,0)\}$ lies in no quadrangle with any of the edges of the form $\{(1,0),(20, i)\}$, for $i \neq 0$, and it follows from part (c) of Lemma 6.9 that all those edges must lie in the same factor of $Y$ as $\{(1,0),(2,0)\}$, say $U$. The same argument holds for the edge $\{(1,0),(7,0)\}$, and so this lies in $U$ as well. Hence $U$ contains all $m+2$ edges incident with the vertex $(1,0)$. By vertex-transitivity and connectivity, all edges of $Y$ lie in $U$, and so $U=Y$. Thus $X(F, m)$ is prime.

Lemma 8.5. For every integer $m \geq 2$ there exists a prime VT graph with arc-type $1+$ $(m+m)$.

Proof. This is very similar to the proof of Lemma 8.4. Again, let $X$ be the graph with arctype $1+(1+1)$ given in Figure 3, but this time take $F$ to be the edge-orbit of $X$ containing the edge $\{1,2\}$ (and the edge $\{1,7\}$ ). Then the thickened $m$-cover $Z=X(F, m)$ of $X$ over $F$ is vertex-transitive, with valency $1+2 m$. Also the edge from $(1,0)$ to $(20,0)$ lies in no quadrangles, which implies immediately that $Z$ is prime. On the other hand, the edge from $(1,0)$ to $(2,0)$ lies in $(2 m-1)^{2}$ quadrangles, and so again the edge from $(1,0)$ to $(2,0)$ cannot lie in the same edge-orbit as the edge from $(1,0)$ to $(20,0)$. Next, $X \backslash F$ is a union of 10 non-incident edges, and hence by part (a) of Theorem 7.6, we find that 
$Z=X(F, m)$ has arc-type $1+(m+m)$ or $2 m+1$. Finally, as before, there are $4 m^{2}$ cycles of length 7 containing the edge from $(1,0)$ to $(2,0)$, and $4 m^{2}$ containing the edge from $(1,0)$ to $(7,0)$, but when two of these cycles differ in only one vertex, it is in the 4 th vertex in the former case, but in the 6 th vertex in the latter case, and so there can be no automorphism of $Z$ taking the $\operatorname{arc}((1,0),(2,0))$ to the $\operatorname{arc}((1,0),(7,0))$. Hence the arc-type of $Z$ is $1+(m+m)$.

Now we consider the marked partition $(1+1)+(1+1)$ of 4 . For this one, we use a quite different construction.

Lemma 8.6. There are infinitely many prime VT graphs with arc-type $(1+1)+(1+1)$.

Proof. For any prime number $p \equiv 1 \bmod 6$, let $G$ be the group $C_{p} \rtimes_{k} C_{6}$, generated by two elements $a$ and $b$ of orders 6 and $p$ such that $a^{-1} b a=b^{k}$, where $k$ is a primitive 6 th root of $1 \bmod p$. Also take $S=\left\{x, y, x^{-1}, y^{-1}\right\}$ where $x=a$ and $y=b a^{2}$, and let $X$ be the Cayley graph $\operatorname{Cay}(G, S)$.

Then $X$ is a 4-valent VT graph, and from the natural action of $G$ by right multiplication, it is easy to see that all edges of the form $\{g, x g\}$ or $\left\{g, x^{-1} g\right\}$ lie in a single edge-orbit, as do all edges of the form $\{g, y g\}$ or $\left\{g, y^{-1} g\right\}$. We now show that these edge-orbits are distinct, and that each gives rise to two distinct arc-orbits, by proving that the stabiliser in $\operatorname{Aut}(X)$ of vertex 1 is trivial.

First, we observe that $0 \equiv 1-\left(k^{2}\right)^{3} \equiv\left(1-k^{2}\right)\left(1+k^{2}+k^{4}\right) \bmod p$, and then since $k^{2} \not \equiv 1 \bmod p$, we have $1+k^{2}+k^{4} \equiv 0 \bmod p$. It follows that

$$
y^{3}=\left(b a^{2}\right)^{3}=b\left(a^{-4} b a^{4}\right)\left(a^{-2} b a^{2}\right)=b b^{k^{4}} b^{k^{2}}=b^{1+k^{4}+k^{2}}=b^{0}=1 .
$$

In particular, every edge of the form $\{g, y g\}$ or $\left\{g, y^{-1} g\right\}$ lies in a 3-cycle (associated with the relation $y^{3}=1$ ). On the other hand, it is easy to see that no edge of the form $\{g, x g\}$ or $\left\{g, x^{-1} g\right\}$ lies in a 3 -cycle, and so $X$ has two distinct edge-orbits, and its edge-type is $2+2$.

Similarly, we note that $0 \equiv 1-\left(k^{3}\right)^{2} \equiv\left(1-k^{3}\right)\left(1+k^{3}\right) \bmod p$ but $k^{3} \not \equiv 1 \bmod$ $p$, so $k^{3} \equiv-1 \bmod p$, and therefore $y x=b a^{3}=a^{3} b^{-1}=a^{-3} b^{-1}=x^{-1} y^{-1}$. Hence the two vertices $x$ and $y^{-1}$ have two common neighbours, namely 1 and $y x$. Also $x y=$ $x(y x) x^{-1}=x\left(x^{-1} y^{-1}\right) x^{-1}=y^{-1} x^{-1}$, and therefore $x^{-1}$ and $y$ have two common neighbours, namely 1 and $x y$. Furthermore, it is an easy exercise to verify that no other two neighbours of 1 have a second common neighbour.

It follows that every automorphism $\alpha$ of $X$ that fixes the vertex 1 must either fix or swap its two neighbours $y$ and $y^{-1}$, and similarly, must fix or swap its two neighbours $x$ and $x^{-1}$. Also if $\alpha$ swaps one pair, then it must also swap the other pair. Hence $\alpha$ either fixes all four neighbours of 1 , or induces a double transposition on them. By vertex-transitivity, the same holds for any automorphism fixing a vertex $v$. Moreover, if the automorphism $\alpha$ fixes one of the arcs incident with the vertex 1 , then it fixes every neighbour $s$ of 1 , and then since it fixes the arc $(s, 1)$, it must act trivially on the neighbourhood of $s$. Then by induction and connectedness, $\alpha$ fixes every vertex of $X$.

Now suppose $\operatorname{Aut}(X) \neq G$, or equivalently, that the stabiliser in $\operatorname{Aut}(X)$ of each vertex is non-trivial. Now if $\beta$ and $\gamma$ are non-trivial automorphisms of $X$ that fix the vertex 1 , then they induce the same permutation $\left(x, x^{-1}\right)\left(y, y^{-1}\right)$ on the four neighbours of 1 , so $\beta \gamma^{-1}$ acts trivially on the neighbourhood of 1 and hence is trivial, giving $\beta=\gamma$. Hence the stabiliser of vertex 1 contains a unique non-trivial automorphism, which must have 
order 2. In particular, $|\operatorname{Aut}(X)|=2|V(X)|=2|G|$, and so $G$ is a normal subgroup of index 2 in $\operatorname{Aut}(X)$. Moreover, the element of $\operatorname{Aut}(X)$ of order 2 stabilising the vertex 1 must normalise $G$, and hence induces an automorphism $\theta$ of $G$, and from what we saw earlier, $\theta$ swaps $x$ with $x^{-1}$, and $y$ with $y^{-1}$. Now $\theta$ takes $a=x$ to $x^{-1}=a^{-1}$, and $b=y a^{-2}=y x^{-2}$ to $y^{-1} x^{2}=\left(b a^{2}\right)^{-1} a^{2}=a^{-2} b^{-1} a^{2}=b^{-k^{2}}$, and so $\theta$ takes $b^{k}$ to $\left(b^{k}\right)^{-k^{2}}=b^{-k^{3}}=b^{-(-1)}=b$. But on the other hand, $b^{k}=a^{-1} b a$, and so $\theta$ takes $b^{k}$ to $a b^{-k^{2}} a^{-1}=b^{-k}$ (since $a^{-1} b^{-k} a=\left(b^{k}\right)^{-k}=b^{-k^{2}}$ ). Thus $b^{-k}=\left(b^{k}\right)^{\theta}=b$, and it follows that $k \equiv-1 \bmod p$, a contradiction.

Hence no such automorphism $\theta$ of $G$ exists, and we find that $\operatorname{Aut}(X)=G$, and that $X$ has arc-type $(1+1)+(1+1)$, as required.

Finally, we show that $X$ is prime, using a similar argument to the one in the proof of Lemma 8.1. If $X \cong X_{1} \square X_{2}$ where $X_{1}$ and $X_{2}$ are relatively prime non-trivial graphs, then by Theorem 6.6 we have $(1+1)+(1+1)=\operatorname{at}(X)=\operatorname{at}\left(X_{1}\right)+\operatorname{at}\left(X_{1}\right)$, which is impossible, since no VT graph has arc-type $(1+1)$. Hence the prime factors of $X$ must be all the same, and so $X$ is a Cartesian product of (say) $k$ copies of a single prime graph $Y$. But then $6 p=|V(X)|=|V(Y)|^{k}$, which is impossible unless $k=1$, since $p$ is a prime number congruent to $1 \bmod 6$. Thus $X$ itself is prime.

Next, we use the first of these graphs (the one with $p=7$ ) to prove the following.

Lemma 8.7. For every integer $m \geq 2$, there exists a prime VT graph with arc-type $(m+$ $m)+(1+1)$.

Proof. Let $X$ be the graph with arc-type $(1+1)+(1+1)$ in Figure 7 , which is also the graph constructed in Lemma 8.6 for $p=7$, and let $F$ be the edge-orbit of $X$ consisting of all the edges that are not contained in a triangle. (These are the edges corresponding to multiplication by the generator $x$ for $G=C_{7} \rtimes C_{6}$.) Now let $Y=X(F, m)$, the thickened $m$-cover of $X$ over $F$. Then $Y$ is vertex-transitive, by Corollary 7.3, and its valency is $2 m+2$. Also $X \backslash F$ is a disjoint union of triangles, so Theorem 7.6 applies, and tells us that all the edges of $Y$ associated with edges of $F$ lie in the same edge-orbit, and all the edges of $Y$ associated with edges of $E(X) \backslash F$ lie in the same edge-orbit. We will show that $Y$ has arc-type $(m+m)+(1+1)$, by proving that these edge-orbits are distinct, and that each gives rise to two arc-orbits.

We do this by showing that every automorphism of $Y=X(F, m)$ induces a permutation of the fibres over $X$, and therefore projects to an automorphism of $X$. It then follows that any automorphism of $Y$ taking an $\operatorname{arc}((v, i),(w, j))$ to an $\operatorname{arc}\left(\left(v^{\prime}, i^{\prime}\right),\left(w^{\prime}, j^{\prime}\right)\right)$ gives rise to an automorphism of $X$ taking $(v, w)$ to $\left(v^{\prime}, w^{\prime}\right)$. Hence if $(v, w)$ and $\left(v^{\prime}, w^{\prime}\right)$ lie in different arc-orbits of $X$, then $((v, i),(w, j))$ and $\left(\left(v^{\prime}, i^{\prime}\right),\left(w^{\prime}, j^{\prime}\right)\right)$ lie in different arcorbits of $Y$, for all $i, j, i^{\prime}, j^{\prime} \in \mathbb{Z}_{m}$.

Observe that the graph $X \backslash F$ is a disjoint union of 14 triangles in $X$, and that there are no other triangles in $X$. Also it is quite easy to see that every triangle in $Y$ is one of the $14 \mathrm{~m}$ triangles of the form $T_{i}=\{(u, i),(v, i),(w, i)\}$ for some triangle $T=\{u, v, w\}$ in $X$ and some $i \in \mathbb{Z}_{m}$, and that these $14 m$ triangles are pairwise disjoint. In particular, since every automorphism takes triangles to triangles, we find that every automorphism of $Y$ preserves the set of edges of $Y$ the form $\{(u, i),(v, i)\}$ with $i \in \mathbb{Z}_{m}$ and $\{u, v\} \in E(X) \backslash F$, and hence also preserves the set of edges of $Y$ of the form $\{(u, i),(v, j)\}$ with $\{u, v\} \in F$. (This also implies that $Y$ is not edge-transitive, so its edge-type is $m+2$.) 
Next, consider what happens locally around a vertex $(u, i)$ of $Y$. This vertex lies in a unique triangle $T_{i}=\{(u, i),(v, i),(w, i)\}$, where $v=y u$ and $w=y^{-1} u$, and also lies in $m$ edges of the form $(r, j)$ and $m$ edges of the form $(s, j)$, for $j \in \mathbb{Z}_{m}$, where $r=x u$ and $s=x^{-1} u$. The other vertices in the fibre over the vertex $u$ have the form $(u, \ell)$ for some $\ell \in \mathbb{Z}_{m}$, and each of these lies at distance 2 from $(u, i)$.

In fact, there are $2 m$ paths of length 2 from each such $(u, \ell)$ to the given vertex $(u, i)$, namely the $m$ paths of the form $((u, \ell),(r, j),(u, i))$ for $j \in \mathbb{Z}_{m}$, and the $m$ paths of the form $((u, \ell),(s, j),(u, i))$, for $j \in \mathbb{Z}_{m}$. On the other hand, from every other vertex at distance 2 from the given vertex $(u, i)$ there are only 1,2 or $m$ paths of length 2 to $(u, i)$. It follows that the stabiliser in $\operatorname{Aut}(Y)$ of the vertex $(u, i)$ preserves the fibre over the vertex $(u, i)$, and therefore $\operatorname{Aut}(Y)$ permutes the fibres over vertices of $X$.

Thus $X(F, m)$ has arc-type $(m+m)+(1+1)$.

Finally, we show that $Y=X(F, m)$ is prime. If $Y \cong Y_{1} \square Y_{2}$ where $Y_{1}$ and $Y_{2}$ are relatively prime non-trivial graphs, then each $Y_{i}$ is vertex-transitive, and by Theorem 6.6 the arc-type of $Y_{1}$ or $Y_{2}$ is $(1+1)$, which is impossible. Hence the prime factors of $Y$ must be all the same, and so $Y$ is a Cartesian product of (say) $k$ copies of a single prime graph $Z$. Also by part (a) of Lemma 6.9, all the edges of a given triangle lie in the same factor, so $Z$ contains a triangle. But now if $k>1$ then some subgraph of $Y$ is a Cartesian product of two triangles, and in the latter, every vertex lies in two distinct triangles, which does not happen in $Y$. Hence $k=1$, and $Y$ itself is prime.

We use yet another construction in the next case, to produce zero-symmetric graphs with arc-type $1+1+1$. Many examples of such graphs are already well known, but we need an infinite family of examples that are prime. A sub-family of the family we use below appears in $[8$, p. 66].

Lemma 8.8. There are infinitely many prime VT graphs with arc-type $1+1+1$.

Proof. Let $G$ be the dihedral group $D_{n}$ of order $2 n$, where $n$ is any integer of the form $2 m-1$ where $m \geq 6$ (so that $n$ is odd and $n \geq 11$ ). Then $G$ is generated by two elements $x$ and $y$ satisfying $x^{2}=y^{n}=1$ and $x y x=y^{-1}$, and the elements of $G$ are uniquely expressible in the form $x^{i} y^{j}$ where $i \in \mathbb{Z}_{2}$ and $j \in \mathbb{Z}_{n}$. (In fact $G$ is the symmetry group of a regular $n$-gon, with the powers of $y$ being rotations and elements of the form $x y^{j}$ being reflections.)

Now define $X$ as the Cayley graph $\operatorname{Cay}\left(G,\left\{x_{1}, x_{2}, x_{3}\right\}\right)$, where $x_{1}=x, x_{2}=x y$ and $x_{3}=x y^{3}$. This graph is vertex-transitive, and since the $x_{i}$ are involutions, it is 3 -valent and bipartite. We show that $X$ is prime and has arc-type $1+1+1$.

The vertices at distance 2 from the identity element are the products of two of the $x_{i}$, which are all distinct: $x_{1} x_{2}=y, x_{1} x_{3}=y^{3}, x_{2} x_{1}=y^{-1}, x_{2} x_{3}=y^{2}, x_{3} x_{1}=y^{-3}$ and $x_{3} x_{2}=y^{-2}$. In particular, $X$ has no 4 -cycles, and hence is prime. Since $X$ is bipartite, it also follows that the girth of $X$ is 6 .

Next, there are 12 paths of length 3 starting from the identity element, but only 7 vertices at distance 3 from the identity element. Indeed it is an easy exercise to show that the coincidences are precisely the following:

$$
\begin{aligned}
x_{1} x_{2} x_{1} & =x_{2} x_{3} x_{2}=x y^{n-1}, & x_{1} x_{2} x_{3}=x_{2} x_{1} x_{2} & =x_{3} x_{2} x_{1}=x y^{2}, \\
x_{1} x_{3} x_{2} & =x_{2} x_{3} x_{1}=x y^{n-2}, & \text { and } \quad x_{2} x_{1} x_{3} & =x_{3} x_{1} x_{2}=x y^{4} .
\end{aligned}
$$


In particular, the edge $\left\{1, x_{1}\right\}$ lies in exactly four cycles of length 6 , namely $\left(1, x_{1}\right.$, $\left.x_{2} x_{1}, x_{1} x_{2} x_{1}, x_{3} x_{2}, x_{2}\right),\left(1, x_{1}, x_{2} x_{1}, x_{3} x_{2} x_{1}, x_{2} x_{3}, x_{3}\right),\left(1, x_{1}, x_{2} x_{1}, x_{3} x_{2} x_{1}, x_{1} x_{2}, x_{2}\right)$ and $\left(1, x_{1}, x_{3} x_{1}, x_{2} x_{3} x_{1}, x_{3} x_{2}, x_{2}\right)$. Similarly, the edge $\left\{1, x_{2}\right\}$ lies in exactly five 6 cycles, and the edge $\left\{1, x_{3}\right\}$ lies in only three. These numbers are different, and it follows that the edges $\left\{1, x_{1}\right\},\left\{1, x_{2}\right\}$ and $\left\{1, x_{3}\right\}$ lie in distinct arc-orbits.

Hence the arc-type of $X$ is $1+1+1$, as claimed.

The next four lemmas deals with the remaining basic arc-types we need.

Lemma 8.9. There exist more than one prime VT graphs with arc-type $1+(1+1)$.

Proof. We have already observed that the zero-symmetric graph on 20 vertices given in Figure 3 has arc-type $1+(1+1)$, and because not every edge is contained in a 4-cycle, it is prime by Corollary 6.8. Some other examples of graphs of arc-type $1+(1+1)$ appear in $\left[8\right.$, p. 39]; these can be described with LCF-codes $[2 k, 2 k,-2 k,-2 k]^{m}$ for $(m, k) \in\{(13,5),(17,13),(25,7),(29,17)\}$, and they are all prime, since they all have edges that are not contained in 4-cycles.

Lemma 8.10. There exists a prime VT graph with arc-type $1+1+(1+1)$.

Proof. The graph on 20 vertices given in Figure 9 has arc-type $1+1+(1+1)$. Now suppose that this graph is not prime. Then since 20 is not a non-trivial power of any integer, the graph must be the Cartesian product of two smaller connected VT graphs that are relatively prime. Then since there are no VT graphs with arc-type $1+1$ or $(1+1)$, it must be a Cartesian product of two connected VT graphs with arc-types 1 and $1+(1+1)$. The former has to be $K_{2}$, and so the other is a VT graph of order 10 with arc-type $1+(1+1)$. But no such graph exists - in fact, the smallest VT graph with arc-type $1+(1+1)$ has 20 vertices. Hence the given graph is prime.

Lemma 8.11. There exists a prime VT graph with arc-type $1+1+1+1$.

Proof. The graph on 16 vertices given in Figure 10 has arc-type $1+1+1+1$. Also this graph cannot be the Cartesian product of two smaller connected VT graphs that are relatively prime, by a similar argument to the one given in the proof of Lemma 8.10, because there is no VT graph of order 8 with arc-type $1+1+1$. (The smallest VT graph with arctype $1+1+1$ has order 18.) Finally, if it is the Cartesian power of some smaller graph, then it has to be the Cartesian square of $C_{4}$ (or the Cartesian 4th power of $K_{2}$, which is isomorphic to $C_{4} \square C_{4}$ ), but this graph is arc-transitive, with arc-type 4 . Hence the given graph is prime.

Lemma 8.12. There exists a prime VT graph with arc-type $(1+1)+(1+1)+(1+1)$.

Proof. Let $X$ be the Cayley graph $\operatorname{Cay}(G, S)$ for the group $G=S L(2,3)$ of all $2 \times 2$ matrices of determinant 1 over $\mathbb{Z}_{3}$, given by the set $S=\left\{x, x^{-1}, y, y^{-1}, x y,(x y)^{-1}\right\}$, where

$$
x=\left(\begin{array}{ll}
1 & 0 \\
1 & 1
\end{array}\right) \quad \text { and } y=\left(\begin{array}{rr}
0 & 1 \\
-1 & 0
\end{array}\right) .
$$

These two elements generate $G$ and satisfy the relations $x^{3}=y^{4}=1$ and $y x^{-1}=(x y)^{2}$, which are defining relations for $G$. This Cayley graph $X$ is 6 -valent, with girth 3 , and it is not difficult to show that its diameter is 3 . 
In the neighbourhood of the identity element 1 in $X$, there is an edge between $y$ and $x y$, and a path of length 3 from $y^{-1}$ to $(x y)^{-1}$ via $x$ and $x^{-1}$, but there are no other edges (between vertices of that neighbourhood). Also the vertices $y$ and $y^{-1}$ have another common neighbour, namely $y^{2}$, and the vertices $x y$ and $(x y)^{-1}$ have another common neighbour, namely $y^{-1} x y\left(=(x y x)^{-1}\right)$, but $y$ and $(x y)^{-1}$ have no other common neighbour, and $y^{-1}$ and $x y$ have no other common neighbour. It follows that the stabiliser in $\operatorname{Aut}(X)$ of the vertex 1 either fixes all its neighbours, or interchanges $y$ with $x y$, and $y^{-1}$ with $(x y)^{-1}$, and $x$ with $x^{-1}$. But the number of neighbours of the vertex $y$ that are at distance 2 from $x$ is 4 , while the number of neighbours of the vertex $x y$ that are at distance 2 from $x^{-1}$ is only 3, so the latter cannot happen, and hence the stabiliser in $\operatorname{Aut}(X)$ of 1 acts trivially on the neighbourhood of 1 . By vertex-transitivity, the same holds at every vertex, and then by induction and connectedness, it follows that the stabiliser of every vertex is trivial.

Thus $\operatorname{Aut}(X)=G$, making $X$ a GRR. Then since the edges $\{1, s\}$ and $\left\{1, s^{-1}\right\}$ lie in the same edge-orbit for each $s \in S$, we find that $X$ has type $(1+1)+(1+1)+(1+1)$.

Finally, $X$ cannot be the Cartesian product of two smaller connected VT graphs that are relatively prime, since there are no VT graphs with arc-type $(1+1)$. Also $X$ cannot be a Cartesian power of some smaller VT graph, since its order 24 is not a non-trivial power of any integer. Hence $X$ is prime.

The graph used in the proof of Lemma 8.12 is shown in Figure 12.

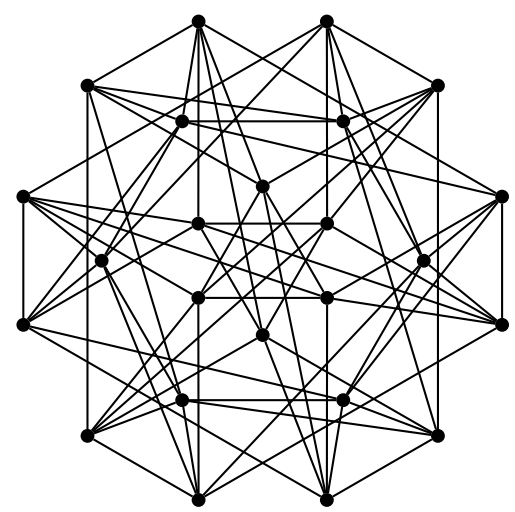

Figure 12: A VT graph with arc-type $(1+1)+(1+1)+(1+1)$, on 24 vertices

\section{Realisability}

We say that a marked partition $\Pi$ is realisable if there exist a vertex-transitive graph with arc-type $\Pi$. Recall that the marked partitions $1+1$ and $(1+1)$ are not realisable (as we explained in the introductory section), and on the other hand, some other marked partitions with few summands are realisable by infinitely many vertex-transitive graphs (as we showed in Section 8).

In this final section we prove that all other marked partitions are realisable. We then find (as a corollary) that all standard partitions except $1+1$ are realisable as the edge-type of a vertex-transitive graph. 
Theorem 9.1. Every marked partition other than $(1+1)$ and $1+1$ is realisable as the arc-type of a vertex-transitive graph.

Proof. Let $\Pi=n_{1}+\cdots+n_{t}+\left(m_{1}+m_{1}\right)+\cdots+\left(m_{s}+m_{s}\right)$ be a marked partition of an integer $d \geq 2$, different from $1+1$ and $(1+1)$. We may assume that $n_{1} \geq \cdots \geq n_{t}$ and $m_{1} \geq \cdots \geq m_{s}$. If $d \leq 4$, then we know from the examples given in Section 5 that $\Pi$ is realisable, and therefore we may assume that $d \geq 5$ when necessary.

We will show how to find a VT graph with arc-type $\Pi$, by taking the Cartesian product of prime graphs with smaller degrees and simpler arc-types, chosen so that the sum of their arc-types is $\Pi$. To do this, we consider separately the two cases where $s=0$ and $t=0$, with a focus on the number of $n_{i}$ or $m_{j}$ that are equal to 1 , respectively, and then we combine these two cases in order to show how to handle all possibilities.

Case (A): $s=0$, with $\Pi=n_{1}+\cdots+n_{t}$.

Let $k$ be the number of $n_{i}$ that are equal to 1 , so that $n_{i}>1$ for $1 \leq i \leq t-k$, and $n_{t}=1$ for $t-k+1 \leq i \leq t$.

If $k=0$, then by Lemma 8.1 we can find $t$ pairwise non-isomorphic prime VT graphs with arc-types $n_{1}, \ldots, n_{t}$, and by Theorem 6.6, their Cartesian product is a VT graph with arc-type $\Pi$.

If $k=1$, we can take the Cartesian product of $t-2$ pairwise non-isomorphic prime VT graphs with arc-types $n_{1}, \ldots, n_{t-2}$, and one prime VT graph with arc-type $n_{t-1}+1$, as given by Lemma 8.3, and again, this is a VT graph with arc-type $\Pi$.

If $k=2$, we can take the Cartesian product of $t-3$ pairwise non-isomorphic prime VT graphs with arc-types $n_{1}, \ldots, n_{t-3}$, one prime VT graph with arc-type $n_{t-2}+1$, and the graph $K_{2}$.

Finally, if $k \geq 3$, we can take the Cartesian product of $t-k$ pairwise non-isomorphic prime VT graphs with arc-types $n_{1}, \ldots, n_{t-k}$, plus

(i) $k / 3$ pairwise non-isomorphic prime VT graphs with arc-type $1+1+1$ taken from Lemma 8.8 , when $k \equiv 0 \bmod 3$, or

(ii) one prime VT graph of type $1+1+1+1$ from Lemma 8.11 , and $(k-4) / 3$ pairwise non-isomorphic prime VT graphs with arc-type $1+1+1$, when $k \equiv 1 \bmod 3$, or

(iii) one copy of $K_{2}$, and one prime VT graph of type $1+1+1+1$, and $(k-5) / 3$ pairwise non-isomorphic prime VT graphs with arc-type $1+1+1$, when $k \equiv 2 \bmod$ 3.

Case (B): $t=0$, with $\Pi=\left(m_{1}+m_{1}\right)+\cdots+\left(m_{s}+m_{s}\right)$.

Let $\ell$ be the number of $m_{j}$ that are equal to 1 , so that $m_{j}>1$ for $1 \leq i \leq s-\ell$, and $m_{j}=1$ for $s-\ell+1 \leq i \leq s$.

If $\ell=0$, then by Lemma 8.2 we can find $s$ pairwise non-isomorphic VT graphs with arc-types $\left(m_{1}+m_{1}\right), \ldots,\left(m_{s}+m_{s}\right)$, and then their Cartesian product is a VT graph with arc-type $\Pi$.

If $\ell=1$, we can take the Cartesian product of $s-2$ pairwise non-isomorphic VT graphs with arc-types $\left(m_{1}+m_{1}\right), \ldots,\left(m_{s-2}+m_{s-2}\right)$, and one prime VT graph with arc-type $\left(m_{s-1}+m_{s-1}\right)+(1+1)$ from Lemma 8.7.

Finally, if $\ell \geq 2$, we can take the Cartesian product of $s-\ell$ pairwise non-isomorphic prime VT graphs with arc-types $\left(m_{1}+m_{1}\right), \ldots,\left(m_{s-\ell}+m_{s-\ell}\right)$, plus 
(i) $\ell / 2$ pairwise non-isomorphic prime VT graphs with arc-type $(1+1)+(1+1)$ from Lemma 8.6, when $\ell$ is even, or

(ii) one prime VT graph of type $(1+1)+(1+1)+(1+1)$ from Lemma 8.12 , and $(\ell-3) / 2$ pairwise non-isomorphic prime VT graphs with arc-type $(1+1)+(1+1)$, when $\ell$ is odd.

Case (C): $s>0$ and $t>0$.

In this case, we can write $\Pi$ as the sum of the marked partitions $\Pi_{1}=n_{1}+\cdots+n_{t}$ and $\Pi_{2}=\left(m_{1}+m_{1}\right)+\cdots+\left(m_{s}+m_{s}\right)$, and we can deal with most possibilities by simply taking a Cartesian product of a VT graph $X_{1}$ with arc-type $\Pi_{1}$ and a VT graph $X_{2}$ with arc-type $\Pi_{2}$. Note that case (A) uses the prime VT graphs produced by Lemmas 8.1, 8.3, 8.8 and 8.11, plus the graph $K_{2}$, while case (B) uses the prime VT graphs produced by Lemmas 8.2, 8.7, 8.6 and 8.12. These prime graphs can be chosen to be pairwise nonisomorphic, and hence pairwise relatively prime, in which case $X_{1}$ and $X_{2}$ are relatively prime, and therefore $X_{1} \square X_{2}$ has arc-type $\Pi_{1}+\Pi_{2}=\Pi$.

All that remains for us to do is to deal with the exceptional situations, namely those where the sum of the $n_{i}$ or the sum of the $m_{j}$ is so small that no suitable candidate can be found for $X_{1}$ or $X_{2}$. There are two exceptional possibilities for $\Pi_{1}$ not covered in case (A), namely 1 and $1+1$, and just one for $\Pi_{2}$ in case $(B)$, namely $(1+1)$.

If $\Pi_{1}=1$, then we can take a Cartesian product of $K_{2}$ with a VT graph produced in case (B), since we are assuming that $d \geq 5$.

If $\Pi_{1}=1+1$, then there are two sub-cases to consider, depending on the number $\ell$ of terms $m_{j}$ that are equal to 1 . If $\ell<s$, then we can adapt the approach taken in case (B) by replacing the prime VT graph of type $\left(m_{1}+m_{1}\right)$ by a prime VT graph of type $1+\left(m_{1}+m_{1}\right)$ from Lemma 8.5, and then also add a single copy of $K_{2}$ as above. On the other hand, if $\ell=s$, so that $\Pi_{2}$ is the sum of $s$ terms of the form $(1+1)$, then we take

(i) two non-isomorphic prime VT graphs with arc-type $1+(1+1)$ from Lemma 8.9, when $s=2$, or

(ii) a prime VT graph of type $1+1+(1+1)$ from Lemma 8.10, and a $2(s-1)$-valent VT graph of type $(1+1)+\cdots+(1+1)$ as found in case (B) when $s \geq 3$.

Finally, if $\Pi_{2}=(1+1)$, again there are two sub-cases to consider, this time depending on the number $k$ of terms $n_{i}$ that are equal to 1 . If $k<t$, then we can adapt the approach taken in case (A) by replacing the prime VT graph of type $n_{1}$ by a prime VT graph of type $n_{1}+(1+1)$ from Lemma 8.4. On the other hand, if $k=t$, so that $\Pi_{1}$ is the sum of $t$ terms all equal to 1 , then we take

(i) a single copy of $K_{2}$ and a prime VT graph with arc-type $1+1+(1+1)$ from Lemma 8.10 , when $t=3$, or

(ii) a prime VT graph of type $1+(1+1)$ and a $(t-1)$-valent VT graph of type $1+\cdots+1$ as found in case (A) when $t \geq 4$.

This completes the proof.

Corollary 9.2. With the exception of $1+1$ (for the integer 2 ), every standard partition of a positive integer is realisable as the edge-type of a vertex-transitive graph. 
Proof. This follows easily from Theorem 9.1. In fact, every such partition $n_{1}+\cdots+n_{t}$ (except $1+1$ ) occurs as both the edge-type and the arc-type of some VT graph with the property that all of its arc-orbits are self-paired.

\section{Acknowledgements}

We would like to thank Primož Potočnik and Gabriel Verret for fruitful discussions about vertex-transitive graphs, and Matjaž Konvalinka and Marko Petkovšek for their advice about partitions. Also we acknowledge the use of MAGMA [2] in constructing examples of graphs with a given arc-type and small valency, as well as in testing various cases. In the course of experimenting, Mathematica and Sage [25] were used as well.

\section{References}

[1] L. Babai and C. D. Godsil, On the automorphism groups of almost all Cayley graphs, European J. Combin. 3 (1982), 9-15, doi:10.1016/S0195-6698(82)80003-6, http: / / dx . doi .org/ $10.1016 / \mathrm{s} 0195-6698$ (82) $80003-6$.

[2] W. Bosma, J. Cannon and C. Playoust, The Magma algebra system. I. The user language, J. Symbolic Comput. 24 (1997), 235-265, doi:10.1006/jsco.1996.0125, http://dx.doi. org/10.1006/jsco.1996.0125.

[3] I. Z. Bouwer, An edge but not vertex transitive cubic graph, Canad. Math. Bull. 11 (1968), 533-535, doi:10.4153/CMB-1968-063-0, http://dx.doi.org/10.4153/ CMB-1968-063-0.

[4] M. F. Capobianco and C. F. Pinzka, Problems and Solutions: Solutions of Elementary Problems: E2055, Amer. Math. Monthly 76 (1969), 194-195, doi:10.2307/2317284, http: / / dx. doi.org/10.2307/2317284.

[5] M. Conder, Trivalent (cubic) symmetric graphs on up to 10,000 vertices, http://www . math. auckland.ac.nz/ conder/symmcubic10000list.txt.

[6] M. Conder and P. Dobcsányi, Trivalent symmetric graphs on up to 768 vertices, J. Combin. Math. Combin. Comput. 40 (2002), 41-63.

[7] M. D. E. Conder and A. Žitnik, Half-arc-transitive graphs of arbitrary even valency greater than 2, European J. Combin. 54 (2016), 177-186, doi:10.1016/j.ejc.2015.12.011, http: // dx.doi.org/10.1016/j.ejc.2015.12.011.

[8] H. S. M. Coxeter, R. Frucht and D. L. Powers, Zero-symmetric graphs : Trivalent graphical regular representations of groups, Academic Press, Inc. [Harcourt Brace Jovanovich, Publishers], New York-London, 1981.

[9] R. M. Foster, The Foster census, Charles Babbage Research Centre, Winnipeg, MB, 1988.

[10] J. L. Gross and T. W. Tucker, Topological graph theory, Wiley-Interscience Series in Discrete Mathematics and Optimization, John Wiley \& Sons, Inc., New York, 1987.

[11] M. Hladnik, D. Marušič and T. Pisanski, Cyclic Haar graphs, Discrete Math. 244 (2002), 137-152, doi:10.1016/S0012-365X(01)00064-4, http://dx.doi.org/10. 1016/S0012-365X(01)00064-4.

[12] D. F. Holt, A graph which is edge transitive but not arc transitive, J. Graph Theory 5 (1981), 201-204, doi:10.1002/jgt.3190050210, http://dx.doi.org/10.1002/jgt. 3190050210 .

[13] W. Imrich and S. Klavžar, Product graphs : Structure and recognition, Wiley-Interscience Series in Discrete Mathematics and Optimization, Wiley-Interscience, New York, 2000. 
[14] W. Imrich and I. Peterin, Recognizing Cartesian products in linear time, Discrete Math. 307 (2007), 472-483, doi:10.1016/j.disc.2005.09.038, http://dx.doi.org/10.1016/j. disc.2005.09.038.

[15] C. H. Li and H.-S. Sim, On half-transitive metacirculant graphs of prime-power order, J. Combin. Theory Ser. B 81 (2001), 45-57, doi:10.1006/jctb.2000.1992, http: / / dx. doi .org/ $10.1006 /$ jctb. 2000.1992 .

[16] A. M. Macbeath, Generators of the linear fractional groups, in: Number Theory (Proc. Sympos. Pure Math., Vol. XII, Houston, Tex., 1967), Amer. Math. Soc., Providence, R.I., pp. 14-32, 1969.

[17] A. Malnič, D. Marušič and P. Potočnik, Elementary abelian covers of graphs, J. Algebraic Combin. 20 (2004), 71-97, doi:10.1023/B:JACO.0000047294.42633.25, http: / / dx . doi . org/10.1023/B: JACO.0000047294.42633.25.

[18] P. Potočnik, P. Spiga and G. Verret, Cubic vertex-transitive graphs on up to 1280 vertices, $J$. Symbolic Comput. 50 (2013), 465-477, doi:10.1016/j.jsc.2012.09.002, http://dx. doi . org/10.1016/j.jsc.2012.09.002.

[19] P. Potočnik, P. Spiga and G. Verret, Bounding the order of the vertex-stabiliser in 3-valent vertex-transitive and 4-valent arc-transitive graphs, J. Combin. Theory Ser. B 111 (2015), 148180, doi:10.1016/j.jctb.2014.10.002, http://dx.doi.org/10.1016/j.jctb.2014. 10.002 .

[20] P. Potočnik, P. Spiga and G. Verret, A census of 4-valent half-arc-transitive graphs and arc-transitive digraphs of valence two, Ars Math. Contemp. 8 (2015), 133-148, http: //amc-journal.eu/index.php/amc/article/view/559.

[21] P. Potočnik and S. E. Wilson, Linking rings structures and tetravalent semisymmetric graphs, Ars Math. Contemp. 7 (2014), 341-352, http: / / amc-journal .eu/index.php/amc/ article/view/311/266.

[22] G. Royle, Transitive graphs, http://staffhome.ecm.uwa.edu.au/ 00013890/ remote/trans/.

[23] G. Sabidussi, On a class of fixed-point-free graphs, Proc. Amer. Math. Soc. 9 (1958), 800-804, doi:10.2307/2033090, http://dx.doi.org/10.2307/2033090.

[24] N. J. A. Sloane, The on-line encyclopedia of integer sequences, http://www. research. att.com/ njas/sequences/.

[25] W. Stein and et al., Sage mathematics software (version 6.4.1), the sage development team, 2014, http: //www. sagemath.org.

[26] W. T. Tutte, A family of cubical graphs, Proc. Cambridge Philos. Soc. 43 (1947), 459-474.

[27] W. T. Tutte, On the symmetry of cubic graphs, Canad. J. Math. 11 (1959), 621-624, doi: 10.4153/CJM-1959-057-2, http://dx. doi.org/10.4153/CJM-1959-057-2.

[28] W. T. Tutte, Connectivity in graphs, Mathematical Expositions, No. 15, University of Toronto Press, Toronto, Ont.; Oxford University Press, London, 1966.

[29] R. Weiss, The nonexistence of 8-transitive graphs, Combinatorica 1 (1981), 309-311, doi: 10.1007/BF02579337, http://dx.doi.org/10.1007/BF02579337.

[30] H. S. Wilf, generatingfunctionology, Academic Press, Inc., Boston, MA, 2nd edition, 1994. 